\title{
Inhibition of ATR prevents macropinocytosis driven retraction of neurites and opposes invasion in GBM
}

Joanna Birch ( $\square$ joanna.Birch@glasgow.ac.uk)

University of Glasgow https://orcid.org/0000-0001-9522-8435

karen Strathdee

University of Glasgow

Katrina Stevenson

University of Glasgow

Sarah Derby

University of Glasgow

Louise Dutton

University of Glasgow

Emily Clough

University of Glasgow

Anna Koessinger

Cancer Research UK Beatson Institute

Lesley Gilmour

University of Glasgow

Ewan McGhee

Beatson Institute

Connor McGarrity-Cottrell

University of Sheffield

Aurelie anderlinden Dibekeme

University of Sheffield

Spencer Collis

University of Sheffield https://orcid.org/0000-0002-7874-1891

Ola Rominiyi

University of Sheffield

\section{Leandro Lembruber Soares}

Cancer Research UK Beatson Institute https://orcid.org/0000-0003-2832-6806

Gergely Solecki

Carl Zeiss Microscopy

Frank Winkler

University Hospital Heidelberg https://orcid.org/0000-0003-4892-6104

Leo Carlin 
Cancer Research UK Beatson Institute https://orcid.org/0000-0001-7172-5234

\section{Gareth Inman}

CRUK Beatson Institute https://orcid.org/0000-0002-6264-4253

\section{Anthony Chalmers}

University of Glasgow https://orcid.org/0000-0002-1746-7278

Jim Norman

CRUK-Beatson Institute https://orcid.org/0000-0002-0098-3014

\section{Ross Carruthers}

Beatson West of Scotland Cancer Centre

\section{Article}

\section{Keywords:}

Posted Date: October 22nd, 2021

DOl: https://doi.org/10.21203/rs.3.rs-967109/v1

License: (c) (1) This work is licensed under a Creative Commons Attribution 4.0 International License. Read Full License 
1 Inhibition of ATR prevents macropinocytosis driven retraction of neurites and opposes invasion in

2 GBM

3 Birch $\mathrm{JL}^{1 *}$, Strathdee $\mathrm{KE}^{1}$, Stevenson $\mathrm{K}^{1}$, Derby $\mathrm{S}^{1}$, Dutton $\mathrm{L}^{1}$, Clough $\mathrm{E}^{1}$, Koessinger $\mathrm{A}^{1,2}$, Gilmour $\mathrm{L}^{1}$,

4 McGhee $\mathrm{E}^{2}$, McGarrity-Cottrell $\mathrm{CL}^{3}$, Vanderlinden $\mathrm{A}^{3}$, Collis $\mathrm{SJ}^{3}$, Rominyi $\mathrm{O}^{3}$, Lemgruber $\mathrm{L}^{4}$, , Solecki

$5 \mathrm{G}^{5}$, Winkler $\mathrm{F}^{6}$, Carlin $\mathrm{LM}^{1,2}$, Inman $\mathrm{G}^{1,2}$, Chalmers $\mathrm{AJ}^{1}$, Norman $\mathrm{JC}^{1,2}$ and Carruthers $\mathrm{R}^{1}$

$6{ }^{1}$ Wolfson Wohl Translational Cancer Research Centre, Institute of Cancer Sciences, University of

7 Glasgow, UK

$8 \quad{ }^{2}$ Beatson Institute of Cancer Research, Glasgow, UK

$9{ }^{3}$ Department of Oncology and Metabolism, The University of Sheffield Medical School, Sheffield, UK

$10{ }^{4}$ University of Glasgow, UK

$11{ }^{5}$ Business Unit Service and Customer Care, Carl Zeiss Microscopy GmbH, 07745 Jena, German

${ }^{6}$ German Cancer Consortium (DKTK), German Cancer Research Center (DKFZ), Heidelberg

*Address for correspondence

Conflict of interest: The authors have declared that no conflict of interest exists 
Glioblastoma (GBM) is the most common and aggressive type of primary brain tumour and remains incurable despite decades of research. GBM are characterised by highly infiltrative growth patterns that contribute to the profound cognitive and neurological symptoms experienced by patients, and to inevitable recurrence following treatment. Novel treatments that reduce infiltration of the healthy brain have potential to ameliorate clinical symptoms and improve survival. Here, we report a novel role of the Ataxia telangiectasia and Rad 3 related kinase (ATR) in supporting the invasive properties of GBM cells through the regulation of macropinocytosis-driven internalisation of integrin adhesion receptors. We demonstrate that inhibition of ATR opposes GBM migration in vitro, and correspondingly reduces infiltrative behaviour in orthotopic mouse models. These results indicate that ATR inhibition, in addition to its use as a radiosensitiser, may be effective in reducing GBM infiltration and its associated symptoms.

\section{Introduction}

Glioblastoma (GBM) is a treatment-refractory brain tumour with a profoundly invasive and migratory phenotype that prevents curative tumour resection resulting in inevitable tumour recurrence. Furthermore, brainstem invasion by GBM cells is a common feature of autopsies, suggesting that infiltration not only contributes to neurological and cognitive dysfunction, but also impacts on mortality and determines clinical outcomes [1]. Consequently, median survival of patients undergoing maximal neurosurgical resection plus adjuvant radiotherapy and chemotherapy remains at only $12-$ 18 months, and only 6 to 8 months for patients with non-resectable tumours [2]. Radiation dose escalation strategies to the tumour bulk have been unsuccessful in improving outcomes whilst increasing the irradiated volume of brain to sterilise invasive microscopic disease is associated with unacceptable toxicity [3] [4]. More detailed understanding of the mechanisms involved in GBM motility and invasion has potential to reveal novel anti-invasive therapies that may enhance the efficacy of existing treatment regimens. Indeed several pre-clinical studies have indicated the value of this approach, but so far none has successfully translated into the clinic [5] [6] [7].

Ataxia telangiectasia and Rad 3 related (ATR) is a phosphatidylinositol 3-kinase (PI3K)-like kinase which is central to the DNA damage and replicative stress response in S phase of the cell cycle where it recruits DNA repair machinery, induces S phase arrest, facilitates replication fork restart and regulates origin firing. Activation of the ATR axis by replication stress has been shown to be a prominent feature 
of glioma stem cells and gliomas in general [8] [9] [10] [11]. However, additional non-canonical roles for ATR have also recently been reported. Cara et al showed that the SCD protein domain targeted by ATR is commonly found in proteins involved in the nervous system, including proteins that regulate vesicle trafficking and cytoskeleton dynamics [12], suggesting a role for ATR beyond the nucleus. This is supported by several studies implicating ATR in cytoplasmic processes including the maintenance of nuclear plasticity to allow interstitial migration [13], regulation of colon tumour cell migration [14] and facilitation of endocytosis and excitatory vesicle recycling at neuronal synapses [15]. The latter observation is of particular interest with regard to GBM cells which share a common neural crest lineage and various morphological features with neuronal cells, including structures that resemble axonal growth cones at the ends of neurite-like projections called tumour microtubes (TMs)[16]. TMs allow interconnectivity between GBM cells and surrounding neuronal cells, forming a functional network that promotes chemotherapy-and radiation- resistance and are associated with invasion into the surrounding brain [17] [18].

Macropinocytosis is a clathrin-independent endocytic process facilitating cellular uptake of high molecular weight, extracellular material and is involved in nutrient uptake, antigen presentation and chemotaxic response [19] [20]. Dysregulated macropinocytosis has been documented in GBM [21, 22]. Intriguingly, macropinocytosis at neuronal axonal growth cones determines repulsive axonal turning and retraction [23] [24]. Furthermore, macropinocytosis is implicated in the rapid trafficking of integrins from focal adhesions to cellular ventral surfaces during growth factor-stimulated cell motility [25] and in facilitating membrane recycling in neural crest cells to drive migration [26]. Strikingly, macropinocytosis appears to be of particular importance in mesenchymal GBM, a subtype that is typically associated with a particularly aggressive and invasive phenotype [22]. These studies provide strong evidence of a role for macropinocytosis in facilitating motility and invasion in GBM.

In this study we report a novel role for ATR kinase in regulating macropinocytic internalisation of integrins and subsequent facilitation of GBM invasion and motility. We demonstrate that this function of ATR is highly sensitive to pharmacological inhibition, resulting in abrogation of GBM invasion both in vitro and in vivo. ATR inhibitors are entering early phase trials as radiation sensitizers and we propose that therapeutic benefit will extend beyond DNA damage potentiation by impacting on invasive potential.

\section{Results}

ATR is found in the cytoplasm and neurite structures of GBM cells, and its expression correlates with invasive potential 
Nuclear ATR activation in response to replication stress has previously been described as a hallmark of GBM cells [9]. Strikingly, high-throughput confocal immunofluorescence imaging of G7 and E2 GBM cells also showed ATR to also be consistently present in the cytoplasm and neurites (Fig 1A (i); Fig S1A). Furthermore, high-throughput imaging and automated quantification of cells subjected to a sublethal dose of radiation (RT; 2Gy), which is known to induce motility in GBM cells [5-7], revealed a significant increase in cytoplasmic ATR (fig 1A (ii)). This indicated a potential role for ATR in regulating GBM invasion. Consistent with this, interrogation of publicly available datasets of mRNA expression in glioma showed that ATR expression increased with tumour grade, being elevated in highly infiltrative Grade IV tumours compared with lower grade gliomas that typically show more contained growth patterns (Fig 1B (i)). Higher ATR expression also correlated with poorer survival (Fig 1B (ii)). Although the reasons behind these observations are likely manifold, the contribution of infiltration to patient survival [1] indicates an intriguing link between ATR and tumour invasion.

Increased ATR expression at the invading edge of human GBM specimens was consistently identified in 3 matched patient tumour core and margin samples by immunohistochemistry (Fig $C(i))$ and by Western blot analysis of cultured Ox5 primary GBM cells isolated from these regions (Fig 1C (i)). Interestingly, this increase in ATR expression corresponded to an increase in length and connectivity of the neurites of Ox5 GBM cells cultured from these different regions (Fig 1C (ii); Fig S1B). These data indicate a potentially clinically relevant association between ATR expression and tumour cell infiltration.

\section{ATR is required to maintain Tumour Microtube (TM) plasticity and support GBM cell migration in} vitro

To investigate the role of cytoplasmic ATR, 3 primary GBM lines, G7, E2 and R15 were treated with the ATR inhibitor, berzosertib (VX970/M6620/VE822). Time-lapse imaging of treated cells revealed a deadhesion/retraction defect at the termini of neurite structures upon ATR inhibition, resulting in their increasing lengthening and fragility, leaving them prone to destabilisation and breakage and ultimately reduced cell motility (Fig 2A; Fig S2; supp. videos 1-2).

To investigate the anti-invasive effect of ATR inhibition further we used single cell tracking to determine the migration speed of sub-confluent G7, E2 and R15 primary cell lines following treatment with increasing doses of berzosertib, with and without radiation (2Gy; Fig 2B). Berzosertib reduced migration speed of all three GBM primary cell lines at concentrations at least 10 -fold below those required to impact on cell viability after 24 hours exposure (Fig. 2B (i), (ii)). For example, the viability IC50 for VE822 in E2 cells was $3.67 \mu \mathrm{M}$ compared to the motility IC50 of $0.34 \mu \mathrm{M}$. This data strongly suggests that ATR has a specific role in regulating the invasive potential of GBM cells that is 
independent of its role in cell survival/DNA damage response. Interestingly, this role does not appear to be linked to the RT- driven motility response that has previously been described [5-7]; although irradiation of $\mathrm{G} 7$ cells further sensitised them to the anti-migratory effects of berzosertib (IC50 $0.26 \mu \mathrm{M}$ vs $96 \mathrm{nM}$ ), this sensitisation was not observed in R15 or E2 cells, suggesting that ATR inhibition effectively inhibits migration in both the presence and absence of radiation.

Berzosertib is a well characterised and highly specific inhibitor of ATR, and its efficacy as an antiinvasive compound at low concentrations suggests that our observations reflect on-target effects of the inhibitor. However, to confirm that the anti-invasive effects of ATR inhibition were not due to an off-target property of berzosertib, we measured migration speed of G7 cells by sub-confluent migration assay and single cell tracking following exposure to siRNAs targeting ATR (Fig 2C). The results demonstrated a significant decrease in migration speed following ATR knockdown, providing confirmation of an on-target effect of ATR inhibition on motility.

Inhibition of ATR opposes GBM cell infiltration in vivo

To confirm the biological relevance of our in vitro migration studies, and to further test the clinical potential of using ATR inhibitors as an anti-invasive strategy, we conducted a series of in vivo studies. Initial pharmacokinetic (PK) studies demonstrated tumour penetration of berzosertib in two different intracranial GBM models (U87MG and the more infiltrative G7) at levels sufficient to inhibit the kinase and induce phenotypic effects (Fig 3A (i) and (ii); Fig S3). Importantly, delivery was enhanced in the tumour compared to the contralateral brain, further enhancing tumour specificity.

Next, we utilised a GFP-labelled S24 GBM primary line in combination with an intracranial window mouse model of GBM that allows intravital imaging of tumours in situ over time (Fig 3B (i)). Intracranial windows were introduced into the skulls of immuno-compromised mice, followed by intracranial injection of GFP-S24 cells. After 3-4 weeks, mice were treated twice with vehicle, followed by multiphoton imaging of tumour cells within the brain. Mice were then treated twice with berzosertib followed by repeat multiphoton imaging (Fig 3B (ii)). Measurement of TM length under control and treatment conditions revealed a significant decrease in length, consistent with the destabilisation and breakage of neurites observed in vitro, and indicative of reduced invasive potential (Fig 3B (iii)). To test this hypothesis further, mice bearing U87MG intracranial tumours were treated with RT in combination with vehicle or berzosertib. Mice were culled at clinical endpoint (symptomatic) and the brains fixed, sectioned and stained for Ki67 to label proliferating GBM cells within the non-proliferative brain tissue. Modest infiltration of tumour cells was observed beyond the tumour margins, as expected following irradiation [5]. Although there was no survival benefit to berzosertib treatment 
(data not shown), a significant decrease in number of infiltrating cells was observed in the berzosertib cohort (Fig 3C (i) and (ii)). Mice were culled with a short time frame (5 days) and no correlation between tumour size and infiltration was found (Fig 3B (iii)). This confirms that the effect of ATR inhibition on tumour cell infiltration is due to loss of invasive potential, and not a function of tumour burden or growth time.

\section{Inhibition of ATR increases cytoplasmic vacuoles}

In addition to the described defect in cell retraction/de-adhesion/cell migration, treatment with berzosertib or siRNA mediated knockdown of ATR increased the appearance of enlarged vacuolar structures within the cytoplasm of GBM cells (Fig 4A(i) and (ii)). We conducted a series of experiments to elucidate the origin of these structures. Autophagy has been demonstrated to modulate cell migration and integrin membrane recycling [27]. To investigate whether a dysregulation of autophagy was underpinning our observations, we undertook autophagic flux assays as previously described [28]. Western blot analysis for processed LC3B from cells treated with/without VE822 and chloroquine indicated no change in the levels of LC3B II, indicating that the observed vacuoles were unlikely to be derived from a block in autophagy (Fig S4A). Indeed, electron microscopy revealed the vacuoles to be universally single membrane bound, inconsistent with double membraned autophagosomes (Fig S4B). This data clearly demonstrates that autophagy is not blocked upon ATR inhibition and does not contribute to the observed ATR dependent phenotype.

Next, we investigated whether these structures could be macropinosomes, which are characteristically large $(0.2-5 \mu \mathrm{m})$ and bound by a single membrane. G7 cells were incubated with a Texas red labelled 70kDa dextran (red) in the presence of berzosertib. Dextrans of this size are predominantly internalised via macropinocytosis, and thus mark any cellular structure that has resulted from this endocytic process. The cells were fixed, stained and subject to confocal imaging (Fig 4B (i)). Indeed, a large proportion of the vacuoles contained dextran, identifying them as macropinosomes. Furthermore, high-throughput confocal imaging and automated analysis of cells treated concurrently with fluorescein labelled 70kDa dextran (green) and DMSO or berzosertib revealed a significant increase in dextran positive macropinosomes in cells treated with berzosertib (Fig 4B (ii)). This accumulation of dextran within the cell body suggests either an increase in macropinocytosis or reduced processing of internalised macropinosomes. Strikingly, immunofluorescence staining revealed a close association between ATR (green) and dextran positive macropinosomes (red), suggesting a direct mechanistic link (Fig. 4C).

Inhibition of ATR causes a block in both active macropinocytosis at the plasma membrane and processing of internalised macropinosomes 
To test whether the increase in cytoplasmic macropinosomes was due to an increase in active macropinocytosis or a block in processing we conducted a pair of timed experiments. In the first instance, cells were treated with either DMSO, berzosertib or an inhibitor of macropinocytosis (5-(NEthyl-N-isopropyl) amiloride; EIPA) for 15 mins or 2 hours prior to the addition of $70 \mathrm{kDa}$ dextran (green). Cells were allowed to internalise dextran for 30 mins before fixing, staining and highthroughput imaging and automated analysis (Fig 5A (i)). Rather than the expected increase in macropinocytosis, we observed a significant dose-dependent decrease in dextran positive macropinosomes in berzosertib pre-treated cells (Fig 5A (ii)), indicating a block in active macropinocytosis at the plasma membrane and mirroring the EIPA effect of blocking macropinocytosis.

With inhibition of active macropinocytosis being observed upon pre-treatment with berzosertib (Fig 5A (ii)), but a clear increase in dextran positive macropinosomes apparent when VE822 and dextran are given concomitantly, a block in the processing of cytoplasmic macropinosomes must also be occurring. To confirm this, the experiment was repeated, allowing cells to internalise dextran for 30 mins in the absence of compound, prior to its removal and addition of DMSO, berzosertib or EIPA for 15 mins (Fig 5B). The results show that turnover of pre-internalised dextran is reduced in a dose dependent manner in cells treated with berzosertib, strongly suggesting that a block in processing is the cause of intracellular dextran accumulation. These effects differed from those of EIPA treatment, suggesting that the processing block is specific to ATR inhibition.

While macropinocytosis has been well characterised in vitro, in vivo studies have proven challenging. The intracranial window model presents an opportunity to bridge this gap. In order to confirm that our observations were not an in vitro artefact, we utilised the window model to look for dextran accumulation in GFP-S24 cells in vivo. Using advanced imaging and processing with Imaris software we were able to confidently detect internalised fluorescent 10kDa dextran within GFP-S24 cells within the mouse brain (Fig 5C (i)). 10kDa dextran was used in this instance to allow multiple subcutaneous injections, and for efficient delivery across the blood-brain barrier. Unlike 70kDa molecules, 10kDa dextran can be internalised by other endocytic processes, however a significant amount is internalised via macropinocytosis [29]. We confirmed via in vitro uptake assays that its endocytosis was similarly impacted by ATR inhibition and thus suitable for our in vivo study (data not shown). We then used this technique to test whether berzosertib caused an accumulation of dextran in vivo by combining vehicle/berzosertib with dextran labelled with two different fluorophores. Mice were initially treated on two consecutive days with vehicle alongside cascade blue-dextran (CB-dex), before multiphoton imaging. They were then treated twice with berzosertib alongside Texas red-dextran (TR-dex), before repeat imaging (Fig 5C (ii)). By changing the colour of the dextran label, we ensured that only dextran internalisation/processing that may have been affected by berzosertib treatment was detected in the 
second imaging session. To control for any variance in fluorophore detection affecting results, the order of administration of dextran colours was switched in one mouse (TR-dex/veh then CB-dex/ berzosertib),and an additional mouse was treated with berzosertib and CB-dex alone. Subsequent colocalization analysis revealed that berzosertib caused an accumulation of dextran in GFP-S24 cells, confirming the biological relevance of our in vitro findings (Fig $5 \mathrm{C}$ (iii).

Together, these data indicate that blocks in both active macropinocytosis and macropinosome processing occur in tandem after ATR inhibition. These results are consistent with time-lapse data from cells treated with berzosertib that show the number of macropinosomes remaining constant over time (Supp Video 2). These observations are reminiscent of previous studies showing that ATR inhibition causes a block in both endocytosis and processing of excitatory vesicles at neuronal synapses [15], and suggest a similar role in endocytosis and vesicle trafficking in GBM cells.

\section{Macropinocytosis is required to internalise integrins at TM growth-cone like structures, allowing TM de-adhesion, retraction and cell migration.}

The parallel observations of a de-adhesion/retraction defect caused by an inability of TMs to release from the extra cellular matrix (ECM), plus a block in macropinocytosis are intriguing, raise the possibility of a mechanistic connection between the two phenomena. To test this theory, we treated

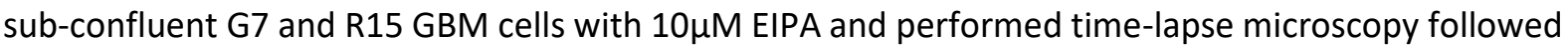
by single cell tracking to measure motility speed. Cells treated with EIPA showed a striking morphological resemblance to cells treated with berzosertib (Fig 1B; Fig 6A), with a similar increase in vacuoles, although these turned over more rapidly than in cells treated with berzosertib, reflecting the observations made in Fig 5B (iii) (Fig 6A (i); Sup video 3). Importantly, EIPA exposed cells showed a similar retraction defect to that resulting from ATR inhibition, leading to a significant reduction in migration speed (Fig 6B (ii), (iii)). These data strongly suggest that macropinocytosis is required for neurite retraction and cell motility in GBM cells.

Macropinocytosis can be utilised by cells to allow bulk trafficking of integrins, which are membrane receptors required for adherence to the ECM. Efficient engagement and release of integrin-mediated adherence is required for efficient cell motility and invasion [30]. Intriguingly, immunofluorescent staining of E2 cells treated with berzosertib revealed enrichment of integrin $\alpha 6$ on the surface of the stalled macropinosomes, both in the cell body (Fig. 7A (i)), and the growth cone like terminus of neurites (Fig. 7A (ii)). We hypothesised that macropinocytosis is required for rapid release of neurite growth cone-like structures through integrin internalisation and hence allow active migration of GBM cells. This hypothesis is supported by previous studies showing the importance of macropinocytosis in regulating this process at neuronal growth cones $[23,24]$. 
To investigate this hypothesis, we performed integrin internalisation assays for $\alpha 5, \alpha 3$ and $\alpha 6$ integrins. Transferrin R (TrnR) is passively, as opposed to actively, internalised and was used as a control. The data in Fig 7B (i)- (iv) clearly indicate that internalisation of all three integrins is reduced upon treatment with Berzosertib, providing further evidence of a role of ATR in regulating integrin internalisation. The reduction is modest, suggesting that internalization may be occurring in a discrete location in the cell.

To investigate this in greater detail, we over expressed GFP-labelled $\alpha 5$ integrin in G7 cells and used super-resolution, time-lapse microscopy to look at $70 \mathrm{kDa}$ dextran uptake at growth cone like structures at the ends of neurites (Fig 8). In control cells we obtained clear evidence that macropinocytosis was occurring at areas of membrane retraction, and that many of the resulting macropinosomes were positive for both GFP- $\alpha 5$ integrin and Texas red labelled dextran (Fig 8A (i)). In addition to a subset of larger macropinosomes that were largely static, we observed a population of highly mobile smaller macropinosomes consistent with observations from studies in neuronal crest cells [26]. Some large endosomal structures were dextran negative, but their size was indicative of macropinosomes which had not internalised dextran.

Importantly, (Fig 8B) we observed that berzosertib downregulated de novo macropinocytosis, with all pre-existing, internalised macropinosomes becoming static, regardless of size. This suggests that the processing defect that causes accumulation of intracellular macropinosomes may be due to defective trafficking of the structures through the cytoplasm, either to be recycled to the membrane or shuttled to the lysosomes for degradation.

These data provide strong evidence to support our hypothesis that ATR is required for effective internalisation of integrins and subsequent de-adhesion and retraction of TMs to enable effective migration in vitro and in vivo.

\section{Discussion}

In this paper we present data that describe a novel, non-canonical role for ATR kinase in facilitating tumour cell motility and invasion via macropinocytic internalisation of integrins and subsequent growth cone de-adhesion. Importantly, we also present evidence for the potential clinical use of ATR inhibitors as anti-invasive therapy. The widespread malignant infiltration observed in GBM is accepted to be a major determinant of the poor clinical outcomes associated with this disease, however a detailed understanding of the key mechanisms involved remains elusive. Abrogation of tumour 
infiltration is a highly desirable clinical target which could improve symptom control and survival in this tumour of unmet need. However, no clinically useful anti-invasive treatments currently exist. Inhibiting invasion has been shown to correlate with increased mouse survival in previous studies using different GBM models $[5,6]$. The absence of a survival benefit in our U87MG experiment may be attributed to the rapidity of growth of the model, as well its comparatively restricted pattern of invasion. A rapid increase in tumour burden and associated 'mass effect', is the major cause of clinical symptoms in this model, with less dependence on infiltration of brain stem and other vital structures that is seen in other GBM models. Nevertheless, our data provide strong evidence for a role of ATR in facilitating GBM invasion within the brain and thus identify a potential therapeutic role of ATR inhibition beyond its established function as a radiosensitiser.

Macropinocytosis is a key process in the bulk trafficking of integrins and plasma membrane to allow active migration and is a prominent feature in $\operatorname{GBM}[21,22,31,32]$. The importance of macropinocytosis in growth cone de-adhesion and neurite retraction/redirection has previously been described in neuronal cells $[23,24]$. Therefore, our observation that this process occurs in GBM cells is perhaps unsurprising, considering their shared lineage, morphology and function. It has been clearly demonstrated in the literature that the TMs of GBM cells share multiple properties with the neurite projection of neuronal cells, including the presence of growth cones, the ability to probe and respond to the local environment through dynamic movement, and even the ability to form functional synapses [16-18]. GBM cells can protrude extraordinarily long TMs into the normal brain, pulling the rest of the cell body behind them as they invade. One can postulate that these leading TMs are sensing the new environment, before committing to invading fully. In this situation, the ability to de-adhere rapidly to allow retraction or redirection in response to positive or negative environmental cues would be advantageous.

The canonical functions of ATR in replication stress and DNA damage signalling are well characterised. However, evidence for biological functions beyond the nucleus are slowly emerging $[12,13,15]$. We demonstrate that ATR is required to mediate internalisation of integrins to allow active migration in GBM cells using clinical samples and state of the art in vitro and in vivo techniques. On first consideration, this observation may be surprising. However, the ability of ATR to trigger cell cycle arrest and modulate kinesins and cytoskeletal dynamics provide a compelling mechanistic link [33] [34] [12]. In addition to its role in bulk internalisation of integrins, macropinocytosis has also been implicated in chemotaxic response, and nutrient sensing $[19,20]$. Placing ATR at the forefront of invading TMs puts it in an ideal position to link nutrient and chemokine sensing via macropinocytosis to the cytoskeleton network and cell cycle. In addition, the freezing effect of ATR inhibition on 
macropinosomes at growth cones that we observed via super resolution microscopy indicates that ATR can modulate kinesins and cytoskeletal dynamics in GBM cells beyond the previously described context of the cell cycle.

ATR inhibitors are currently in development in early phase clinical trials and have been the subject of intense interest due to their potentiation of DNA damage in combination with conventional cytotoxic agents and radiation in a range of tumour sites. In the context of neuro-oncology, the ATR/replication stress response axis is a particularly appealing target. Treatment resistance in GBM appears to be driven by a subpopulation of glioma stem cells with high levels of DNA replication stress and ATR activation and we and others have shown potent radiosensitisation by ATR inhibitors in vitro. Our novel finding of an anti-invasive effect of ATR inhibition suggests that the clinical benefits of this approach could be far reaching and extend beyond radiosensitisation. It could be envisaged that ATRi could be employed as a neoadjuvant strategy to promote regression of invasive tumour edge prior to surgery and irradiation as well as in an adjuvant or 'maintenance' manner to reduce or prevent malignant infiltration into vital neural structures. A prolonged period of neo adjuvant anti-invasive therapy with ATRi may be a particularly attractive strategy in managing patients with lower grade gliomas, who often undergo pre-planned 'elective' tumour resection.

In summary we present a novel role for ATR in regulating GBM invasion which has significant relevance for clinical translation. We predict that this previously unappreciated function of ATR will lead to unexpected clinical benefits from therapeutic strategies combining ATR inhibition with radiotherapy.

\section{Materials and Methods}

\section{Transmission Electron microscopy}

Samples were fixed in $2.5 \%$ glutaraldehyde, $4 \%$ paraformaldehyde, in $0.1 \mathrm{M}$ cacodylate buffer, $\mathrm{pH} 7.2$; washed in $0.1 \mathrm{M}$ cacodylate buffer, $\mathrm{pH} 7.2$ and post-fixed in $1 \%$ OsO4 for 1 hour. After several washes in the same buffer, the samples were en bloc stained with $0.5 \%$ uranyl acetate in water for 30 minutes. Afterwards, samples were washed with water, dehydrated in ascending acetone series and resin embedded. Ultrathin sections (50nm thick) were collected and imaged on a JEOL 1200 Transmission electron microscope (JEOL, Japan) operating at 80kV.

In Silico Analysis of ATR expression from public datasets 
Expression of ATR and correlation to patient survival (Kaplan -Meier survival analysis) was performed using the publically available RNA sequencing dataset from the Chinese Glioma Genome Atlas (CGGA) project via the gliovis platform: http://gliovis.bioinfo.cnio.es/.

\section{Derivation and maintenance of primary GBM cell lines}

Primary GBM cell lines E2, G7, and R15 were derived from resected tumors and maintained as described previously [35] [36], approved by the local regional Ethics Committee (LREC ref 04/Q0108/60) in compliance with the UK Human Tissue Act 2004 (HTA License ref 12315). Cell lines were utilized for 10 passages from thaw prior to being discarded and were tested for the presence of mycoplasma using the Lonza MycoAlert (LT07-318) assay every 3 months. Cell lines were cultured on Matrigel-coated plates $(0.23 \mathrm{mg} / \mathrm{L}$ in AdDMEM, Life Technologies) in serum-free AdDMEM supplemented with $20 \mathrm{ng} / \mathrm{mL}$ EGF, $10 \mathrm{ng} / \mathrm{mL}$ FGF, 0.5\% B27 supplement, and $0.5 \% \mathrm{~N} 2$ supplement (all Life Technologies). The commercially available U87MGluc2 line (Caliper Life Sciences) was cultured in MEM EBSS (Life Technologies), 10\% FBS, 1\% L-glutamine, 1 \% NEAA (Life Technologies), and 1\% NaPyruvate (Life Technologies). In vitro irradiation was performed using an Xstrahl RX225 radiation cabinet (195 kV X-rays, dose rate $1.39 \mathrm{~Gy} /$ minute).

\section{In vitro immunofluorescence}

For confocal imaging sub-confluent GBM cells were plated on coverslips coated with Matrigel and incubated at $37^{\circ} \mathrm{C}$ for 24 hours. For high-throughput imaging, $1 \times 10^{4}$ cells were plated on 96 well black sided plates (Perkin Elmer) precoated with Matrigel. Cells were incubated with anti-ATR (23HCLC ThermoFisher) or anti-integrin a6 (CD49F 55734 BD Bioscience) antibodies overnight at $4^{\circ} \mathrm{C}$ followed by incubation with secondary conjugated antibodies, DAPI and HCS Cell Mask Deep Red Stain (ThermoFisher H32721) or Texas red-X Phalloidin (ThermoFisher T7471). For confocal imaging nuclei were counterstained with Vectashield mount containing DAPI. Images were acquired using either a Zeiss LSM 780 or 880 confocal microscope and analyzed using Zen 2012 (Zeiss). For High-throughput analysis, images were captured using the Opera Phenix High-content Screening system and analysed using Columbus Image Analysis software (Perkin Elmer).

\section{Super resolution microscopy}

$0.5 \times 10^{5} \mathrm{G} 7$ cells were plated on glass bottomed $35 \mathrm{~mm}$ dishes pre coated with $5 \mu \mathrm{g} / \mathrm{ml}$ fibronectin (ThermoFisher) and allowed to establish for 24hours. $1 \mu \mathrm{g}$ of plasmid containing $\alpha 5$-GFP was then transiently transfected into the cells using SuperFect transcription reagent (Qiagen). After 24 hours, 
mins before addition of either DMSO or Berzosertib. Timelapse, super resolution imaging was performed using Zeiss 880 Airyscan (SR mode) with a Plan-Apochromat 40x/1.3 Oil objective and images analysed using Imaris software. Cell were maintained at $37^{\circ} \mathrm{C}, 5 \% \mathrm{CO} 2$ in an incubation chamber for the duration of imaging.

\section{Cell viability}

Cell viability was carried out using CellTiter-Glo according to the manufacturer's protocol (Promega). Briefly, cells were plated in matrigel coated 96 well plates and incubated with incremental concentrations of Berzosertib. Cells were irradiated with 2Gy 1 hour after addition of drug and incubated for a further 23 hours prior to detection of luminescence (Promega GLOMAX).

\section{SiRNA transfection}

Subconfluent G7 cells were transfected with 30 pmol siRNA targeting ATR or control (Dharmacon: cat no J-003202-19-002 Lot no: 190724) using RNAiMAX reagent (Invitrogen 13778-030) and plated into 6 well plate then incubated for $48 \mathrm{hrs}$ prior to imaging or protein extractions.

\section{Sub confluent migration assays}

Sub confluent migration assays were performed as previously described [5]. For G7 and E2 lines, $0.5 \mathrm{x}$ $10^{5}$ cells were plated per well of a 12 well plate and allowed to establish overnight before treatment and time-lapse microscopy performed using a Nikon Z6010. For the R15 line, $0.5 \times 10^{4}$ were plated per well of a 24 well plate and imaging acquired using an IncucyteS3 (Sartorius). Images were acquired every 15 mins and cell speed measured via single cell tracking using ImageJ.

\section{Autophagic flux assay}

Cells were plated at sub confluent density in $10 \mathrm{~cm}$ petri dishes and incubated for 24 hours. DMSO, VE822 $1 \mu \mathrm{M}$ and/or chloroquine 10 $\mu \mathrm{M}$ were added to media and cells further incubated until lysis and harvesting at the appropriate timepoint. Cell lysates were subject to Western blotting with LC3B antibody (Cell Signaling 2775), as described previously (ref Carruthers abrogation ATM paper).

\section{Animal experiments}

Animal experiments were performed under the relevant home office licence and in accordance with ARRIVE guidelines. All experiments had ethical approval from the University of Glasgow under the Animal (Scientific Procedures) Act 1986 and the EU directive 2010. Mice were maintained in 
individually ventilated cages with environmental enrichment. Mice used in the intracranial window experiments were housed separately following surgery to prevent damage to the implanted ring.

\section{Intracranial window experiments}

Male NMRI-Foxn $1^{n u}$ mice $(n=5)$ were used for these experiment using an adaptation of a surgical procedure described by Winkler et al, 2004 [37]. Custom made titanium rings and frames for surgery and imaging were supplied by VetTech. 2- 3 weeks after window implantation, mice were injected with 30,000 S24-GFP cells and tumours allowed to develop for a further 2-3 weeks.

Mice were given either vehicle (10\% vitamin E) or Berzotersib $(50 \mathrm{mg} / \mathrm{kg}$ ) by oral gavage according to the schedules described in the results section. $200 \mu \mathrm{l}$ of $10 \mathrm{mg} / \mathrm{ml} 10 \mathrm{kDa}$ dextran was administered subcutaneously. Imaging was undertaken using a Zeiss880 multiphoton microscope using W PlanApochromat 20x/1.0 objective and subsequently analysed using Imaris software. Z-stacks were taken to a depth of $60-100 \mu \mathrm{m}$, at $1 \mu \mathrm{m}$ intervals. GFP and TR-dex were imaged with $890 \mathrm{~nm}$ laser, $C B$ dex with $800 \mathrm{~nm}$. Multiple fields were taken each imaging session, and included individually, or as means as shown by the overlayed plots in Fig 3B and 5B. Statistical analysis = paired student $\mathrm{T}$ Test.

\section{Intracranial tumour experiments}

Female CD1 nude mice were orthotopically injected with $1 \times 10^{5}$ U87MG or G7 cells as previously described [5]. For PK studies, tumours were allowed to establish for 3 weeks (U87MG) or 10 weeks (G7) before dosing with either vehicle (10\% vitamin E) or $60 \mathrm{mg} / \mathrm{kg}$ Berzosertib and mice culled at the indicated time points. Tumors were sub-dissected and fresh-frozen specimens of tumour and contralateral hemispheres sent for PK analysis (Vertex). For the phenotypic study, tumours were allowed to establish for 12 days before treatment. Brain irradiation was performed on an XStrahI Small Animal Radiation Research Platform (SARRP) as previously described [5]. Mice received $5 \times 3 G y$ on alternate days and were dosed via oral gavage with either vitamin $\mathrm{E}$ or $60 \mathrm{mg} / \mathrm{kg}$ Berzosertib daily for 10 days. Formalin-fixed, paraffin-embedded sections were stained for Ki67, and scanned using a Hamamatsu Nanozoomer Slide scanning machine with Leica SlidePath Slide imaging software.

\section{References}

1. Drumm, M.R., et al., Extensive brainstem infiltration, not mass effect, is a common feature of end-stage cerebral glioblastomas. Neuro Oncol, 2020. 22(4): p. 470-479.

2. Stupp, R., et al., Radiotherapy plus concomitant and adjuvant temozolomide for glioblastoma. N Engl J Med, 2005. 352(10): p. 987-96. 
3. Wegner, R.E., et al., National trends in radiation dose escalation for glioblastoma. Radiat Oncol J, 2019. 37(1): p. 13-21.

4. Walker, M.D., et al., Evaluation of BCNU and/or radiotherapy in the treatment of anaplastic gliomas. A cooperative clinical trial. J Neurosurg, 1978. 49(3): p. 333-43.

5. Birch, J.L., et al., A Novel Small-Molecule Inhibitor of MRCK Prevents Radiation-Driven Invasion in Glioblastoma. Cancer Res, 2018. 78(22): p. 6509-6522.

6. Edalat, L., et al., BKK+ channel blockade inhibits radiation-induced migration/brain infiltration of glioblastoma cells. Oncotarget, 2016. 7(12): p. 14259-78.

7. Kegelman, T.P., et al., Inhibition of radiation-induced glioblastoma invasion by genetic and pharmacological targeting of MDA-9/Syntenin. Proc Natl Acad Sci U S A, 2017. 114(2): p. 370375.

8. Yazinski, S.A. and L. Zou, Functions, Regulation, and Therapeutic Implications of the ATR Checkpoint Pathway. Annu Rev Genet, 2016. 50: p. 155-173.

9. Carruthers, R.D., et al., Replication Stress Drives Constitutive Activation of the DNA Damage Response and Radioresistance in Glioblastoma Stem-like Cells. Cancer Res, 2018. 78(17): p. 5060-5071.

10. Qiu, Z., N.L. Oleinick, and J. Zhang, ATR/CHK1 inhibitors and cancer therapy. Radiother Oncol, 2018. 126(3): p. 450-464.

11. Lecona, E. and O. Fernandez-Capetillo, Targeting ATR in cancer. Nat Rev Cancer, 2018. 18(9): p. 586-595.

12. Cara, L., et al., The ATM-and ATR-related SCD domain is over-represented in proteins involved in nervous system development. Sci Rep, 2016. 6: p. 19050.

13. Kidiyoor, G.R., et al., ATR is essential for preservation of cell mechanics and nuclear integrity during interstitial migration. Nat Commun, 2020. 11(1): p. 4828.

14. Pires, I.M., et al., Targeting radiation-resistant hypoxic tumour cells through ATR inhibition. $\mathrm{Br}$ J Cancer, 2012. 107(2): p. 291-9.

15. Cheng, A., et al., ATM and ATR play complementary roles in the behavior of excitatory and inhibitory vesicle populations. Proc Natl Acad Sci U S A, 2018. 115(2): p. E292-E301.

16. Jung, E., et al., Emerging intersections between neuroscience and glioma biology. Nat Neurosci, 2019. 22(12): p. 1951-1960.

17. Osswald, M., et al., Brain tumour cells interconnect to a functional and resistant network. Nature, 2015. 528(7580): p. 93-8.

18. Venkataramani, V., et al., Glutamatergic synaptic input to glioma cells drives brain tumour progression. Nature, 2019. 573(7775): p. 532-538.

19. King, J.S. and R.R. Kay, The origins and evolution of macropinocytosis. Philos Trans R Soc Lond B Biol Sci, 2019. 374(1765): p. 20180158.

20. Lim, J.P. and P.A. Gleeson, Macropinocytosis: an endocytic pathway for internalising large gulps. Immunol Cell Biol, 2011. 89(8): p. 836-43.

21. Colin, M., et al., Dysregulation of Macropinocytosis Processes in Glioblastomas May Be Exploited to Increase Intracellular Anti-Cancer Drug Levels: The Example of Temozolomide. Cancers (Basel), 2019. 11(3).

22. Seguin, L., et al., Macropinocytosis requires Gal-3 in a subset of patient-derived glioblastoma stem cells. Commun Biol, 2021. 4(1): p. 718.

23. Kabayama, H., et al., Syntaxin $1 B$ suppresses macropinocytosis and semaphorin $3 A$-induced growth cone collapse. J Neurosci, 2011. 31(20): p. 7357-64.

24. Kolpak, A.L., et al., Negative guidance factor-induced macropinocytosis in the growth cone plays a critical role in repulsive axon turning. J Neurosci, 2009. 29(34): p. 10488-98.

25. Gu, Z., et al., Integrins traffic rapidly via circular dorsal ruffles and macropinocytosis during stimulated cell migration. J Cell Biol, 2011. 193(1): p. 61-70. 
26. Li, Y., et al., Macropinocytosis-mediated membrane recycling drives neural crest migration by delivering F-actin to the lamellipodium. Proc Natl Acad Sci U S A, 2020. 117(44): p. 2740027411.

27. Tuloup-Minguez, V., et al., Autophagy modulates cell migration and beta1 integrin membrane recycling. Cell Cycle, 2013. 12(20): p. 3317-28.

28. Mrschtik, M., et al., DRAM-3 modulates autophagy and promotes cell survival in the absence of glucose. Cell Death Differ, 2015. 22(10): p. 1714-26.

29. Li, L., et al., The effect of the size of fluorescent dextran on its endocytic pathway. Cell Biol Int, 2015. 39(5): p. 531-9.

30. Hamidi, H. and J. Ivaska, Every step of the way: integrins in cancer progression and metastasis. Nat Rev Cancer, 2018. 18(9): p. 533-548.

31. Caswell, P.T., S. Vadrevu, and J.C. Norman, Integrins: masters and slaves of endocytic transport. Nat Rev Mol Cell Biol, 2009. 10(12): p. 843-53.

32. De Franceschi, N., et al., Mutually Exclusive Roles of SHARPIN in Integrin Inactivation and NFkappaB Signaling. PLoS One, 2015. 10(11): p. e0143423.

33. Fan, G., et al., The ATM and ATR kinases regulate centrosome clustering and tumor recurrence by targeting KIFC1 phosphorylation. Nat Commun, 2021. 12(1): p. 20.

34. Lamm, N., et al., Nuclear F-actin counteracts nuclear deformation and promotes fork repair during replication stress. Nat Cell Biol, 2020. 22(12): p. 1460-1470.

35. Fael Al-Mayhani, T.M., et al., An efficient method for derivation and propagation of glioblastoma cell lines that conserves the molecular profile of their original tumours. J Neurosci Methods, 2009. 176(2): p. 192-9.

36. Ahmed, S.U., et al., Selective Inhibition of Parallel DNA Damage Response Pathways Optimizes Radiosensitization of Glioblastoma Stem-like Cells. Cancer Res, 2015. 75(20): p. 4416-28.

37. Winkler, F., et al., Kinetics of vascular normalization by VEGFR2 blockade governs brain tumor response to radiation: role of oxygenation, angiopoietin-1, and matrix metalloproteinases. Cancer Cell, 2004. 6(6): p. 553-63. 
A

(i)

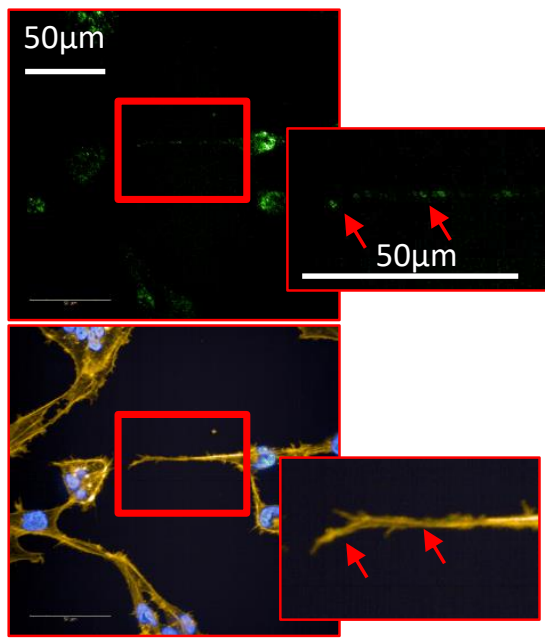

$B_{\text {(i) }}$

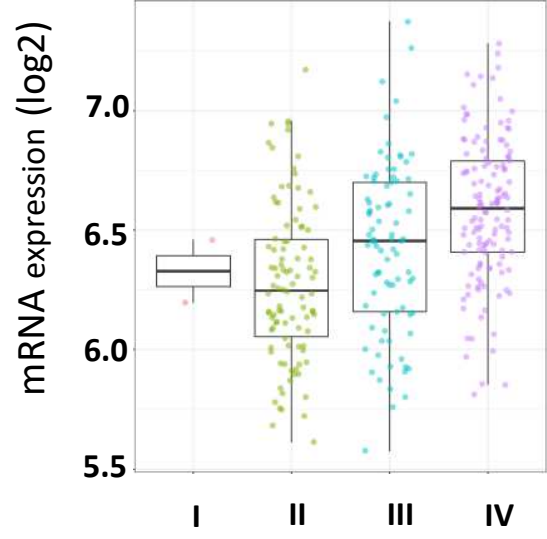

$C$ (i)

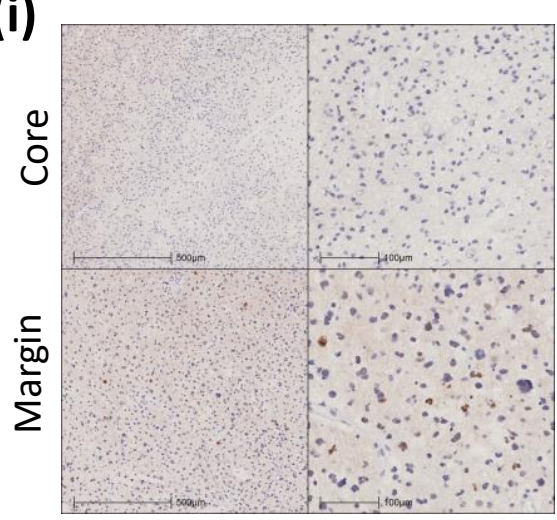

(ii)

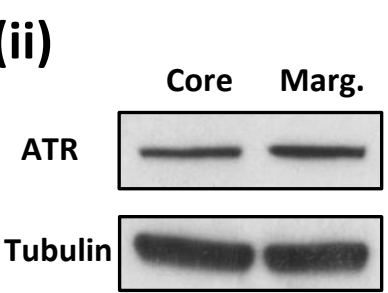

(ii)
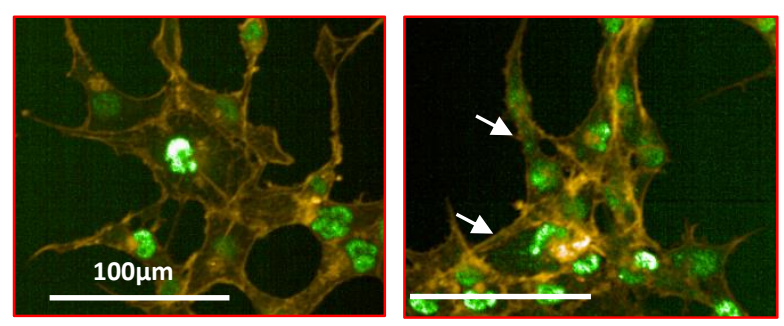

(ii)
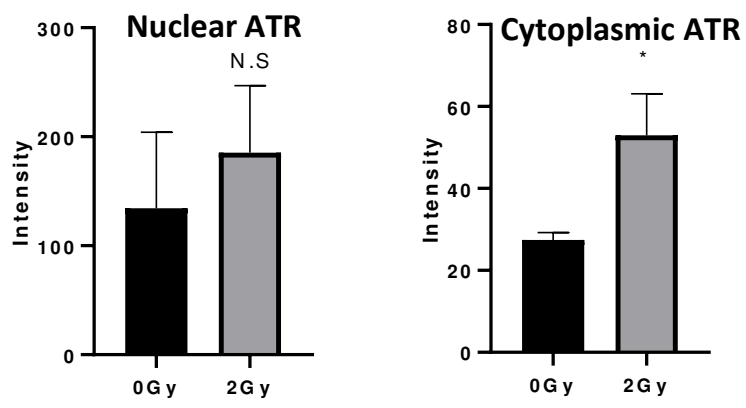
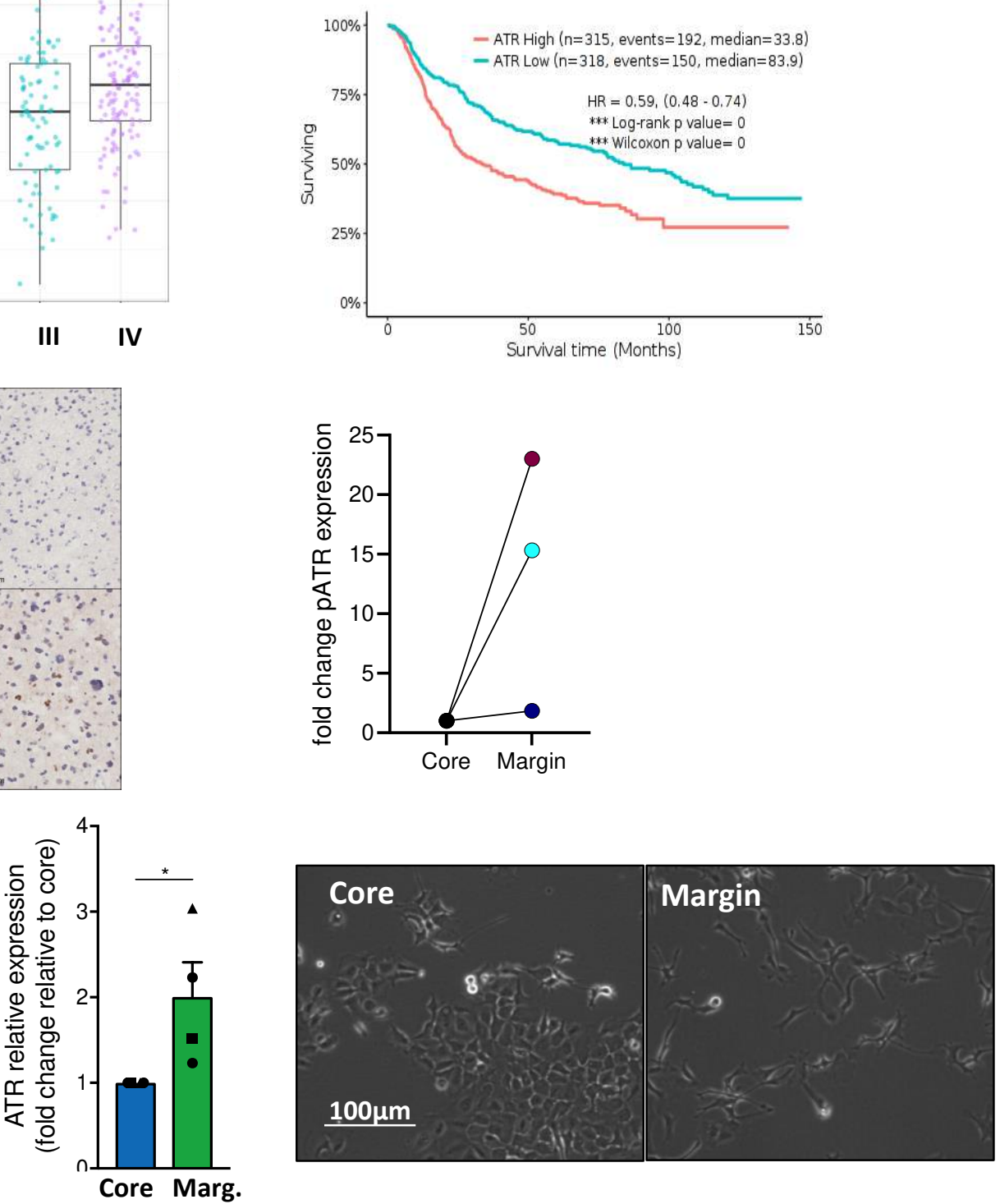
Figure 1: ATR is found in the cytoplasm of GBM cells and correlates with invasive potential A) (i)

ATR is found in both the nucleus and cytoplasm of GBM cells, including distribution along neuritelike projections (red arrows). (ii) Sub-lethal radiation dose causes an increase in cytoplasmic ATR. G7 cells were irradiated with 2Gy and fixed after 6 hours and stained for actin (yellow) and ATR (green) followed by high-throughput imaging. Data from 3 biological repeats, 2000-4000 cells imaged per condition each repeat. Bars are mean \pm -SEM. High-throughput imaging at 40x (A) and 20x (D) BlueDAPI; Green- ATR; Yellow- Actin. B) ATR expression correlates with both (i) glioma grade and poorer patient survival (ii). C) Increased pATR expression can be found in the invasive tumour margin versus the tumour core in patient samples. (i) Core and margin samples were taken from 3 different patients, stained for pATR and scored by a neuropathologist for positive cells and the fold change between core and margin plotted. (ii) ATR expression is increased in primary Ox5 GBM cells derived from the tumour margin compared to matched cells from the core and correlates with increased neurite number and length. Western blot quantified from 4 independent biological repeats.

Statistical analysis using student's T Test. ${ }^{*} p<0.01,{ }^{* * *} p<0.001$ 


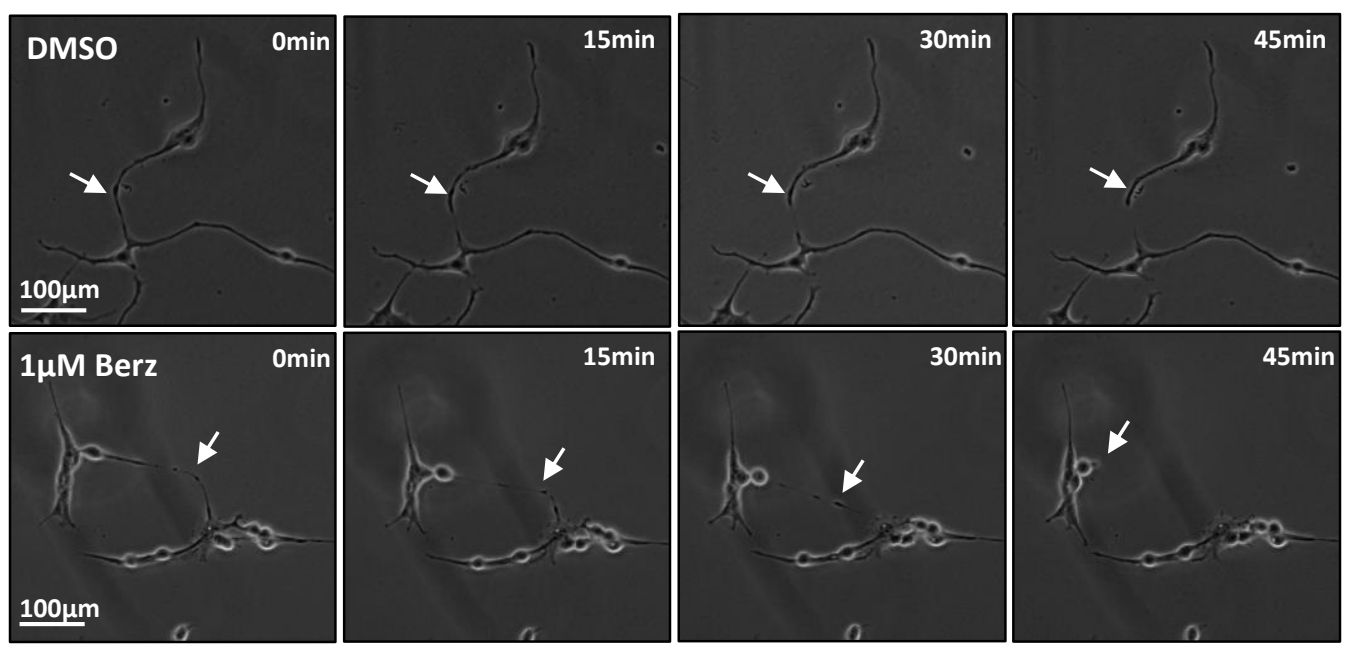

B (i) Motility

G7

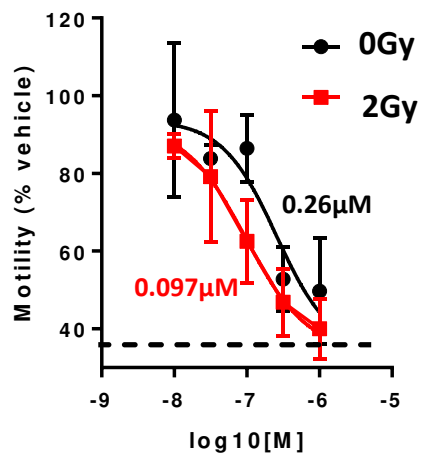

(ii) Viability
R15

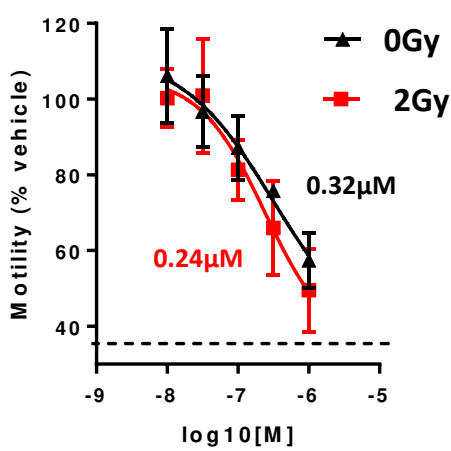

E2

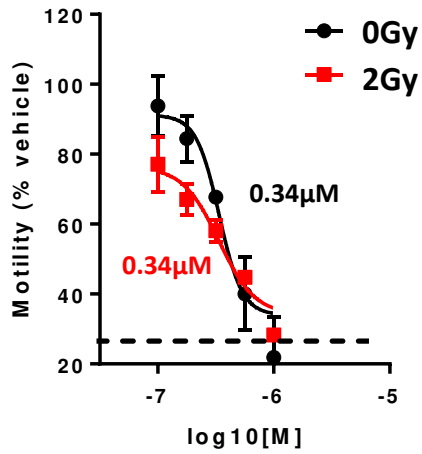

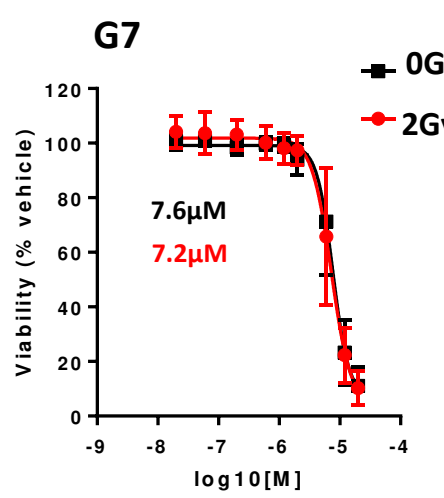

R15

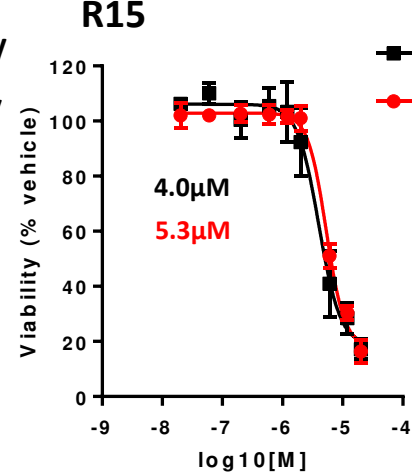

E2

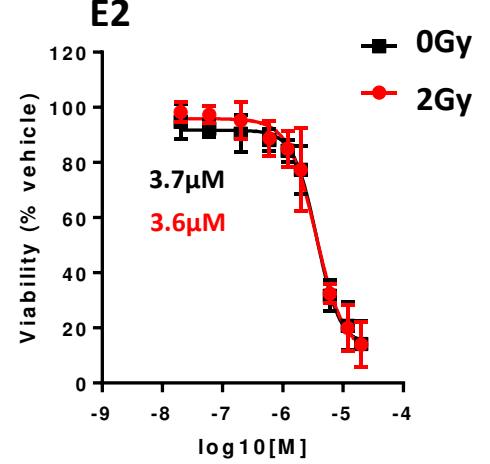

C
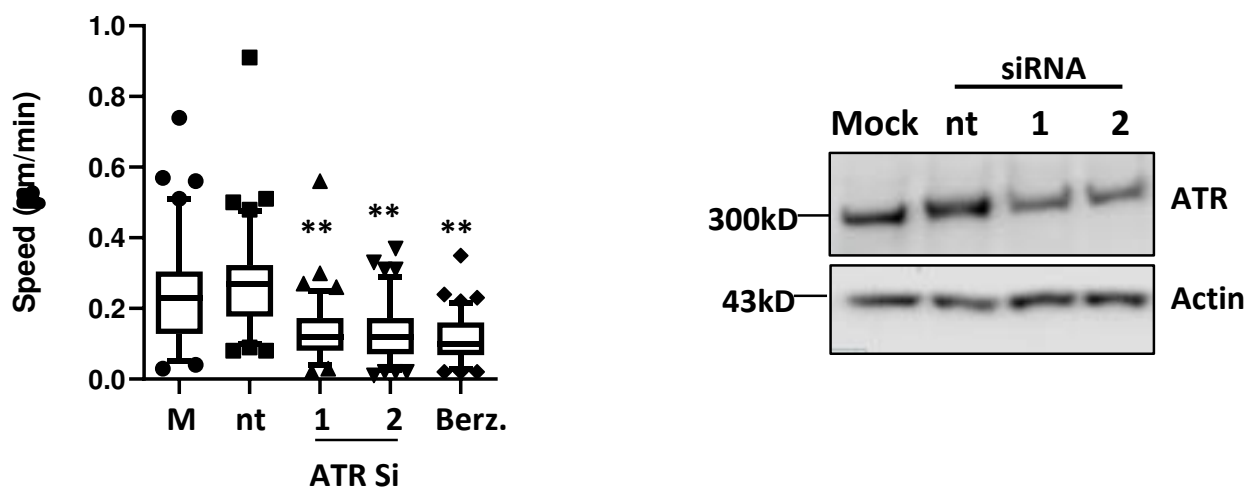
Figure 2: Inhibition of ATR reduces invasive potential in vitro A) Inhibition of ATR induces a neurite retraction defect, causing an aberrant lengthening, followed by breakage and loss of neurite structures in G7 cells. Timelapse following 24 hours of Berz./DMSO exposure. Arrows indicate the termini of neurites. B) Irradiated (2Gy) and non-irradiated GBM cells (G7, R15 and E2) show a dose dependent reduction in migration speed with ATR inhibition (i), at sub-lethal concentrations of inhibitor (iii). Cells were incubated with DMSO/ Berzosertib for $24 \mathrm{hrs}$ followed by irradiation and timelapse microscopy and single cell tracking or viability assay. Data from 3 biological repeats, baseline speed calculated by tracking of $>20$ non-migratory cells. IC50 shown in black (no RT) and red (RT). C) RNAi of ATR inhibits migration speed of $\mathrm{G} 7$ cells. Cells were treated with siRNAs targeting ATR or $1 \mu \mathrm{M}$ Berzosertib followed by timelapse microscopy and single cell tracking. Data from 3 biological repeats, ${ }^{* *} p<0.0001$. Efficient knock down of ATR was confirmed by western blot. 
A

Birch et al; Fig. 3

(i) U87MG

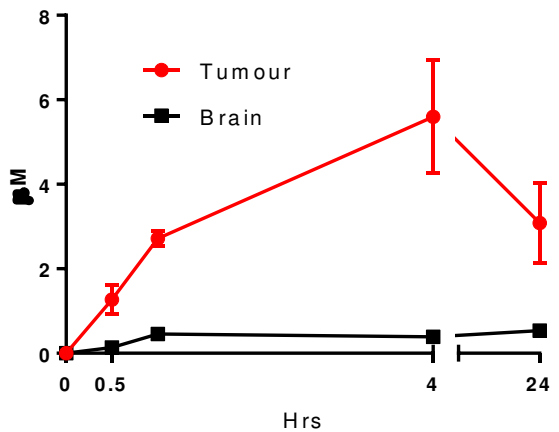

B

(i)

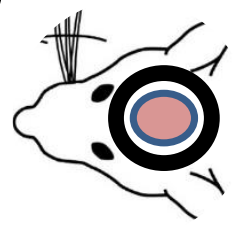

(ii) $\mathrm{G7}$

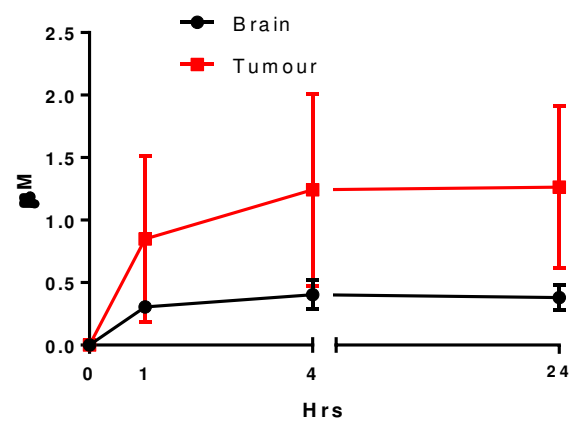

(ii)

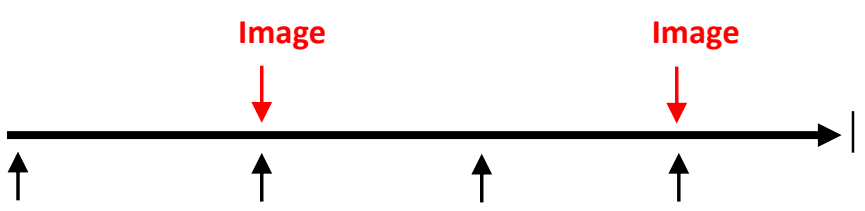

Day 1

Vehicle
Day 2

Vehicle
Day 3

Berz
Day 4

Berz

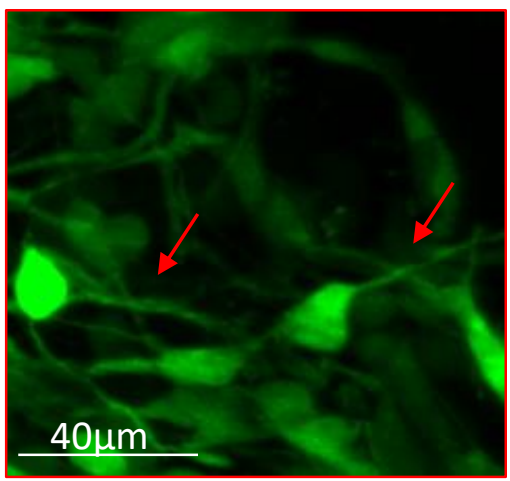

(i)
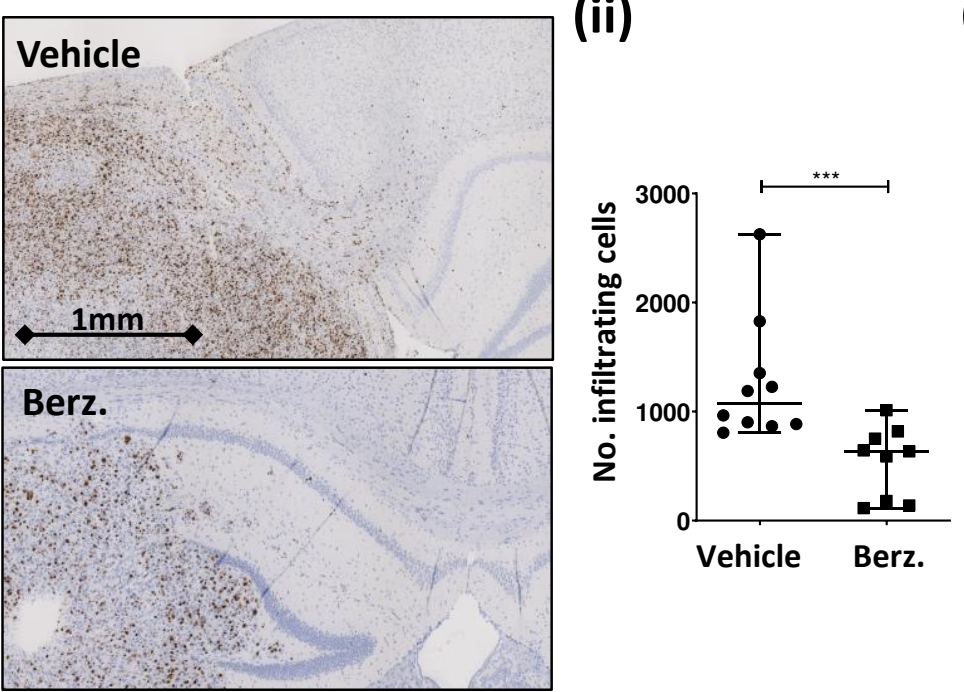

(iii)
Berz.

(ii)

(iii)
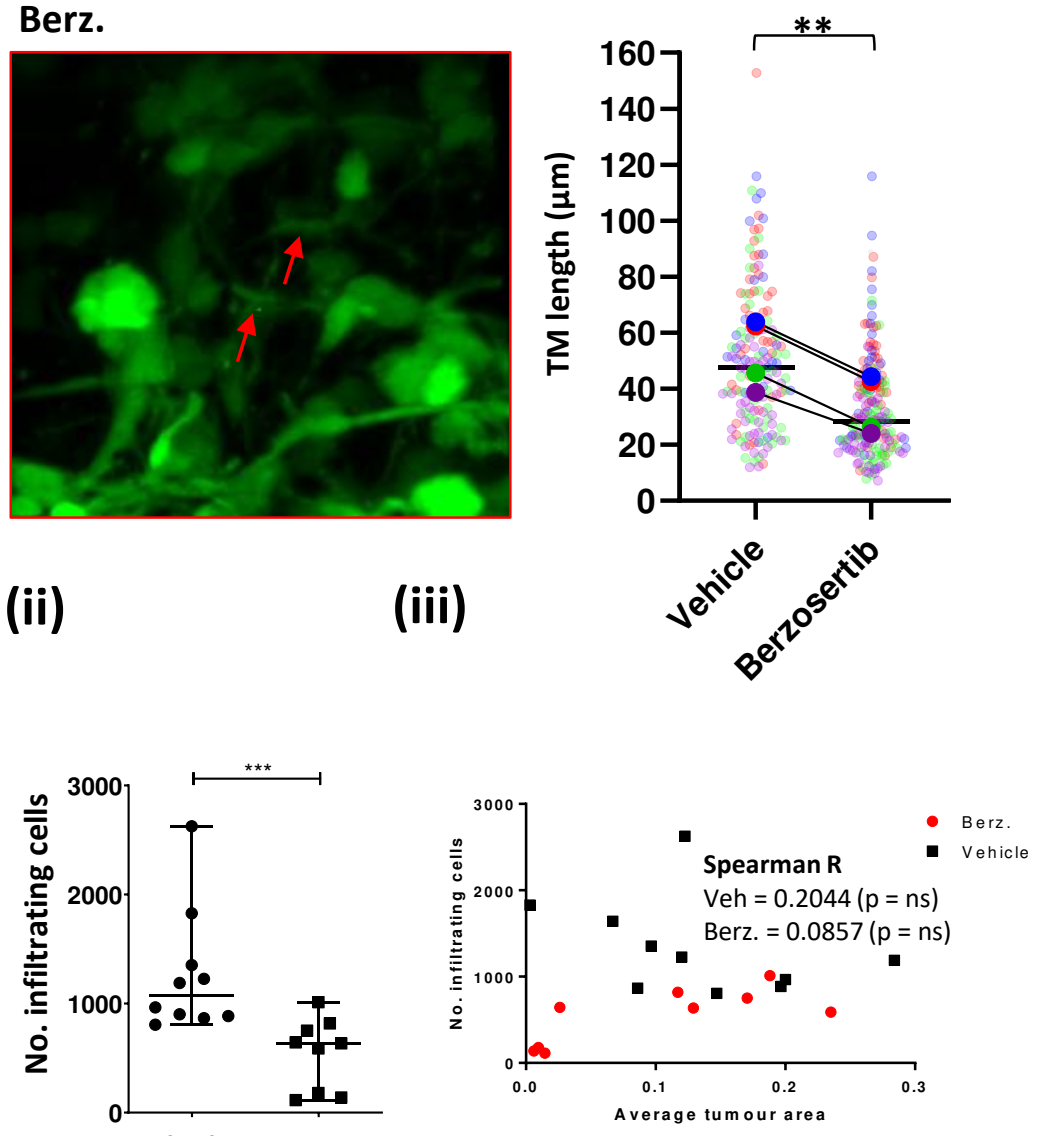
Figure 3: Inhibition of ATR reduces invasive potential in vivo. A) Pharmacokinetic (PK) analysis of Berzosertib delivery to U87MG (i) and G7 (ii) intracranial tumours showed enhanced delivery of compound to the tumour compared to the normal brain (contralateral hemisphere) at biologically relevant levels. B) (i) A longitudinal, intracranial window model of GBM was used to measure the morphological response of S24-GFP cells to ATR inhibition (Berzosertib). Mice were dosed and imaged according to the schedule in (ii). Tumour microtube (TM) length was measured in multiple images per mice and image data plotted individually (small points) or as mean for each mouse (large points), colour coded for each mouse. Arrows indicate TM structures. $\mathrm{N}=5$, one mouse only received Berz, ** $p>0.001$. C) Mice bearing U87MG intracranial tumours were treated with fractionated whole brain radiotherapy concomitant with daily doses of vehicle $(n=9)$ or Berz. $(n=10)(i)$. Coronal brain sections were stained for Ki67 to label GBM cells and the number of infiltrating cells plotted for each animal (ii). (iii) To control for potential discrepancies in tumour size accounting for differential infiltration levels both parameters were plotted against each other. No correlation was observed. Statistical analysis using student's TTest. ${ }^{* * *} p<0.001$. 


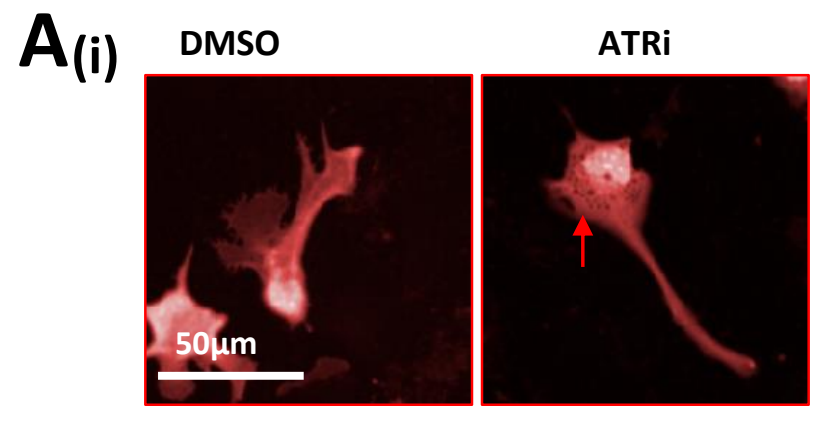

(ii)

Birch et al; Fig. 4

$B_{\text {(i) }}$

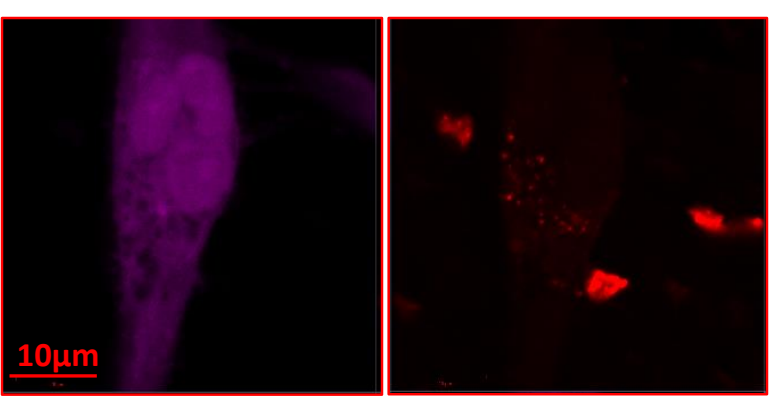

(ii)
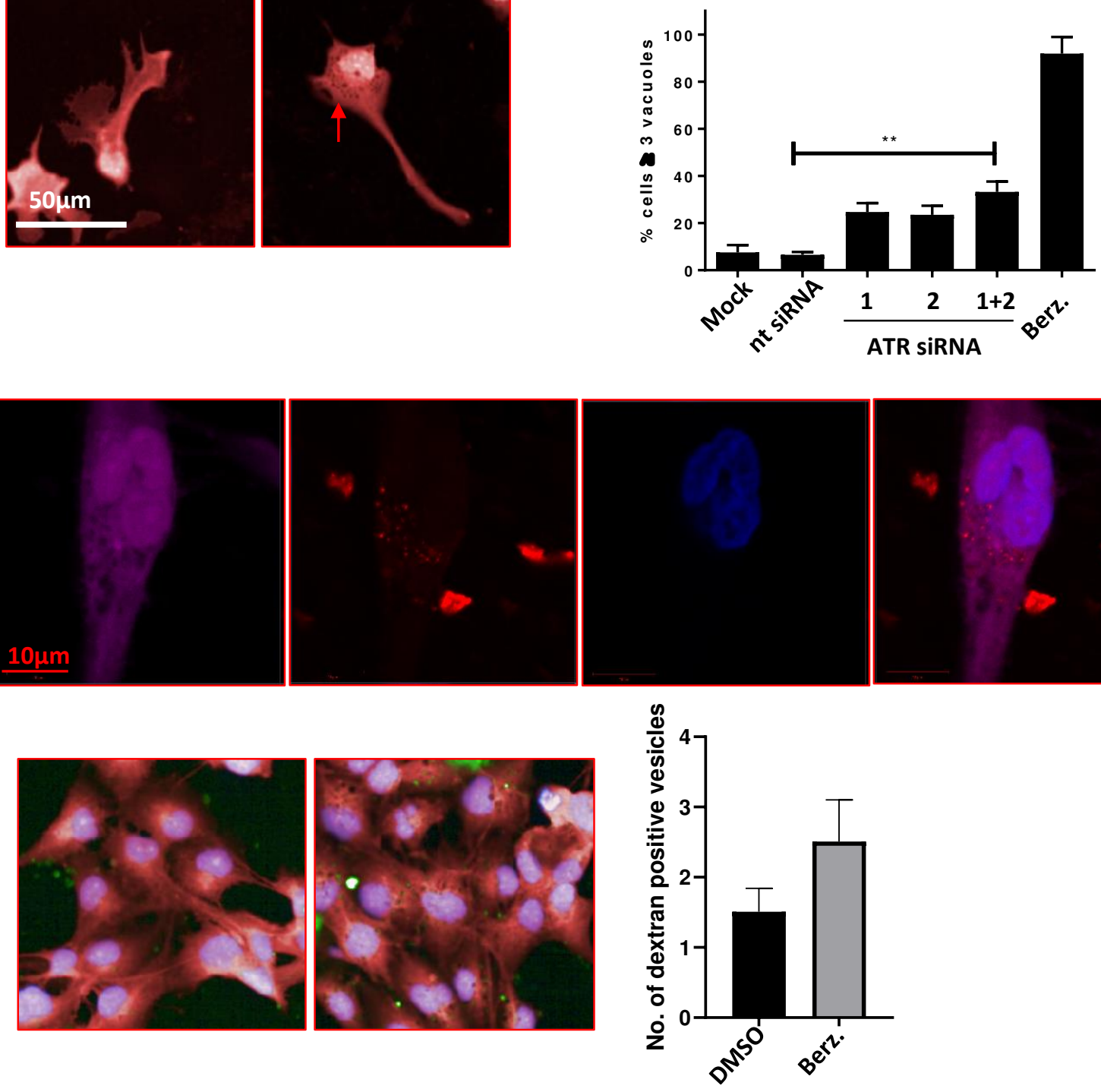

C

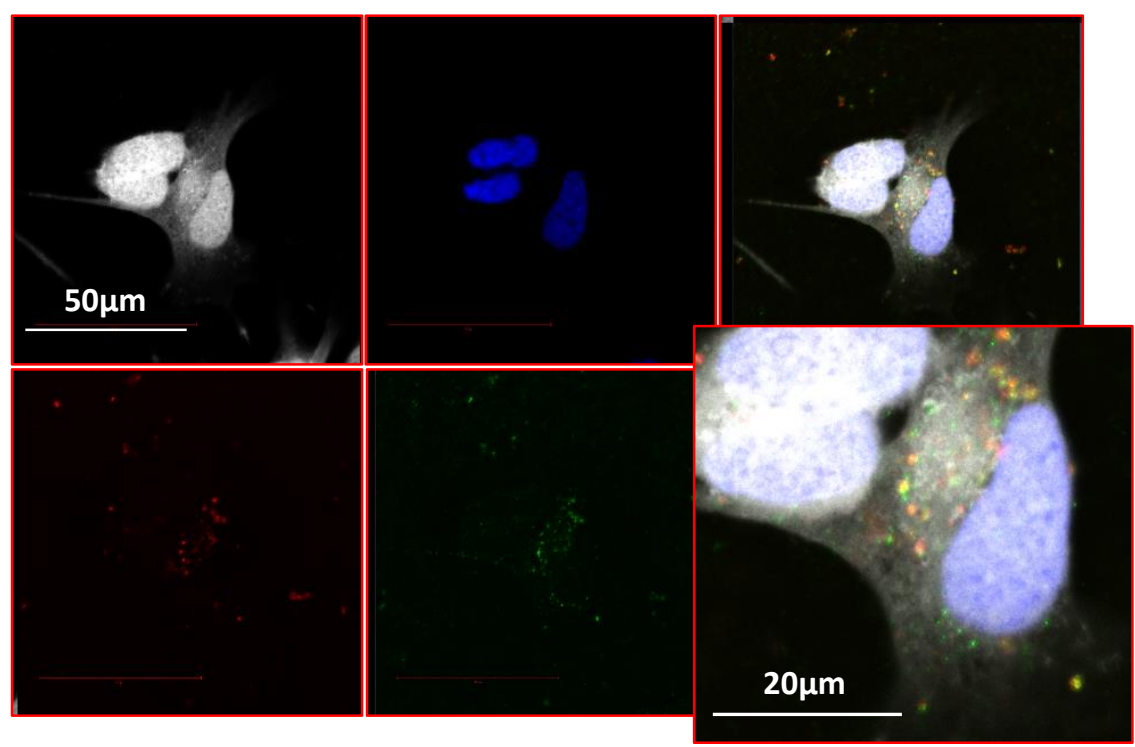


Figure 4: Inhibition of ATR causes an accumulation of macropinosomes in the cell body. A) An accumulation of large vacuoles (red arrow) following either treatment with ATR inhibitor or RNAi of ATR in GBM cells was observed, quantified in (ii). B) (i) Uptake of 70kDa TR-dex into vacuoles. Red = TR-dex, purple $=$ whole cell stain (WCS), blue = DAPI. (ii) Cells were incubated with DMSO/ Berzosertib alongside fluorescein labelled dextran (green) dextran for 30 mins before fixing and quantification of dextran positive vesicles using high throughput imaging. $>4000$ cells quantified per condition. Statistical analysis using student's TTest. ${ }^{*} p<0.05$. C) E2 cells were incubated with 70kDa dextran (red) and $1 \mu \mathrm{M}$ Berzosertib, before fixing and staining for ATR (green), WCS (white) and DAPI (blue) and confocal imaging. 
A

(i)
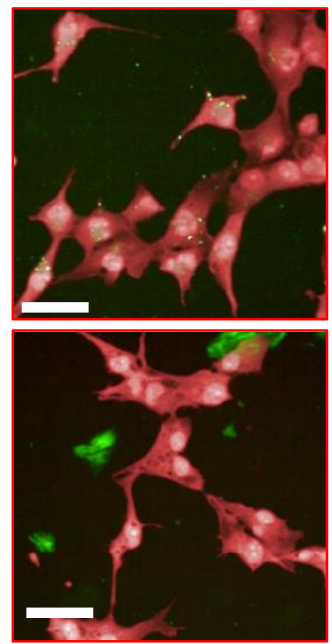

B

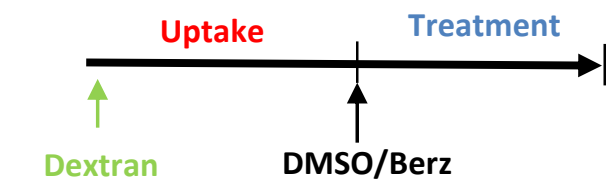

(ii)
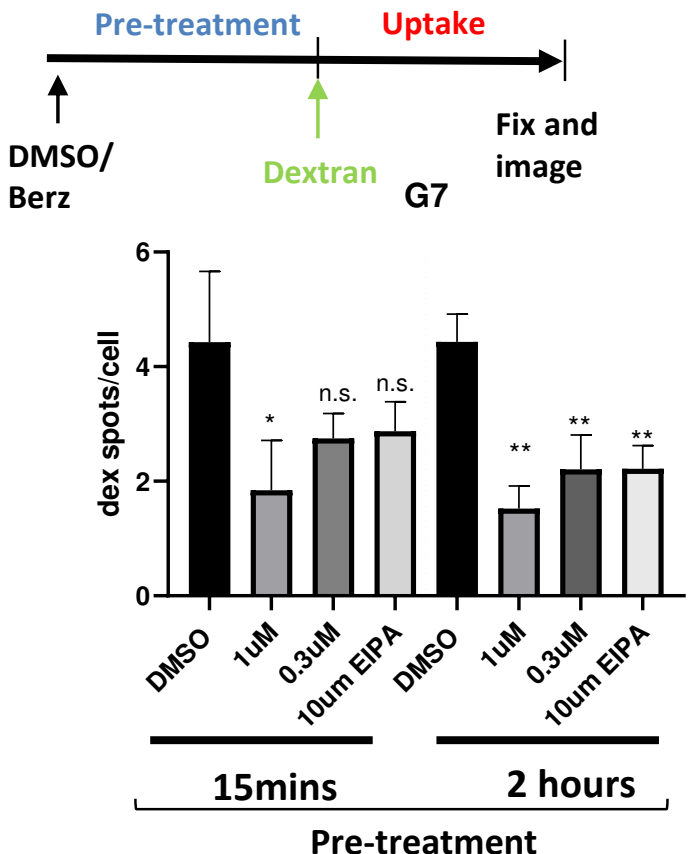

Fix and

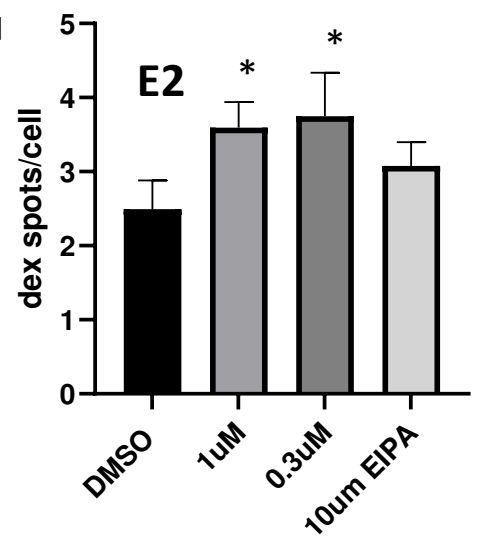

(i)
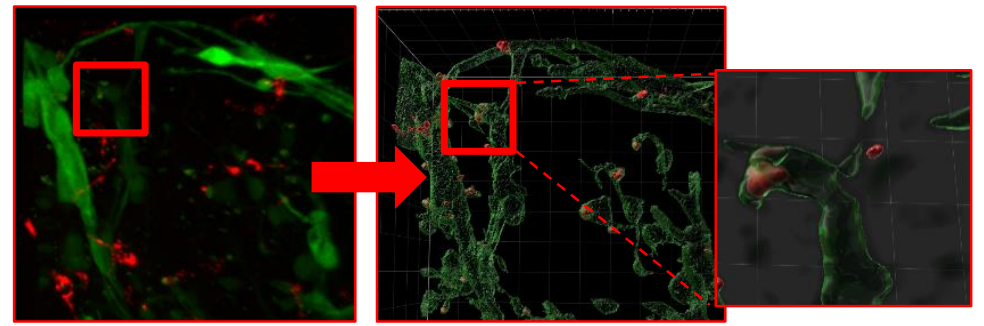

(ii)

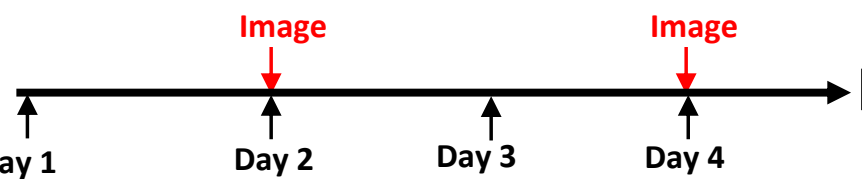

Veh + Dex1 Veh+Dex 1

Berz+ Dex2

Berz+ Dex2 (iii)

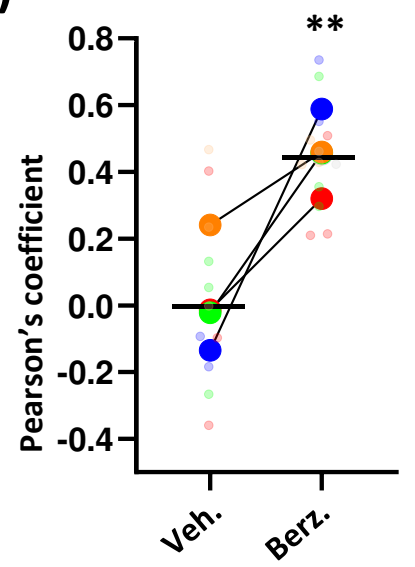


Figure 5: ATR inhibition causes a block in both de novo macropinocytosis, and processing of internalised macropinosomes, leading to an accumulation in vitro and in vivo. A) Pre-treatment of G7 cells with Berzosertib before addition of 70kDa dextran leads to a reduction in dextran uptake indicating a reduction in active macropinocytosis. Cells were incubated with DMSO, Berzosertib or EIPA for 15 mins or 2 hours before incubation with labelled dextran. B) Inhibition of ATR leads to a block in macropinosome processing. G7 or E2 cells were pre-incubated with dextran to allow uptake before addition of DMSO, Berzosertib or EIPA. Data represents 3 biological repeats, $2000-4000$ cells quantified via high throughput imaging per condition per repeat. ${ }^{*} p<0.05 ;{ }^{*} p<0.01$, n.s $=$ not significant. C) Treatment of mice with ATRi Berzosertib in an intracranial window model causes an accumulation of labelled dextran in vivo. (i) Multiphoton imaging and processing was used to detect uptake of fluorescent dextran in to GFP-S24 cells in the mouse brain. (ii) Mice were treated with DMSO and Cascade blue labelled dextran followed by imaging, then treated with Berzosertib and Texas red labelled dextran followed by second imaging. (iii) Pearson's coefficient was calculated under each treatment condition for each mouse to estimate colocalization of dextran with S24-GFP cells using Imaris colocalization tool. $\mathrm{N}=5$ (one mouse received only Berz plus CB-Dex), > 3 images per imaging session per mouse, image data plotted individually (small points) or as mean for each mouse (large points), colour coded for each mouse. ${ }^{* *} p<0.01$, Students TTest 
Birch et al; Fig. 6

A

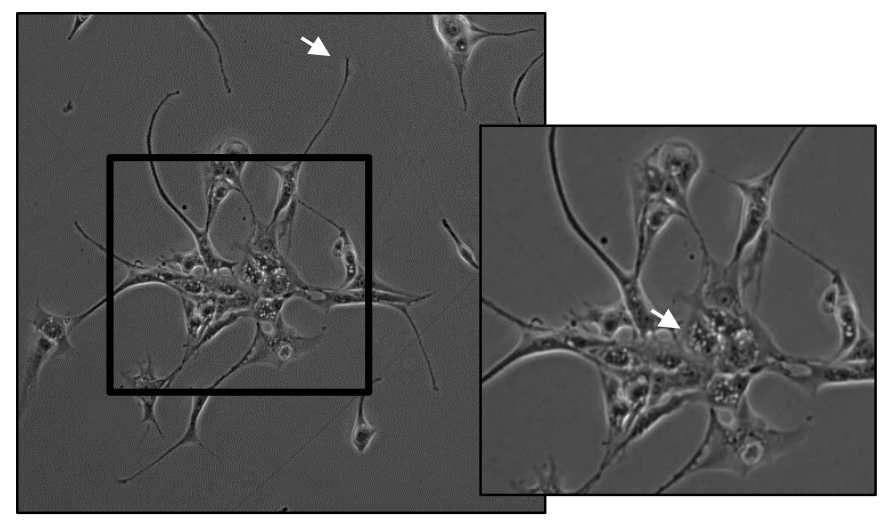

B
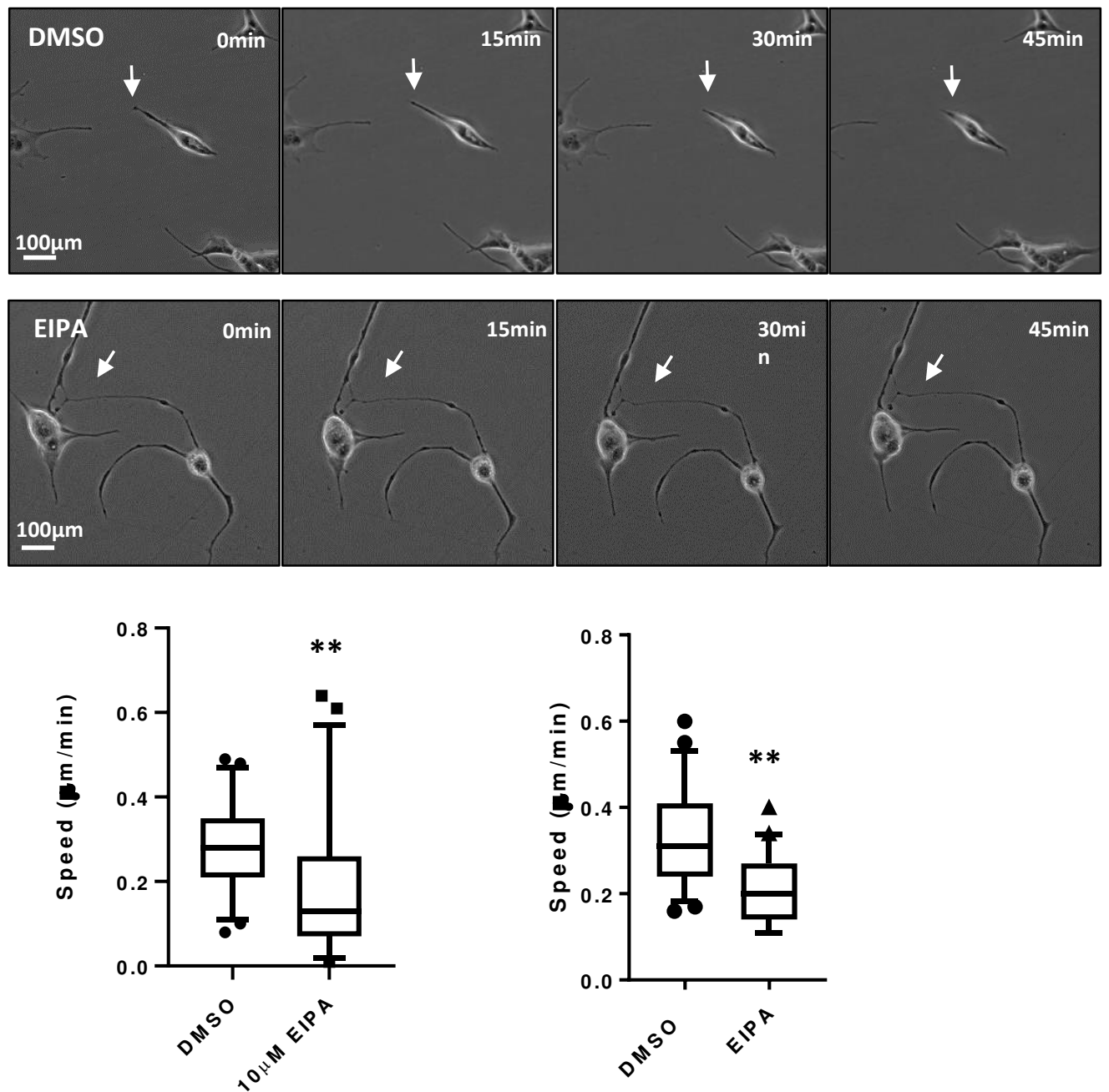
Figure 6: Inhibition of macropinocytosis causes a morphological and subsequent migration defect in

GBM cells. A) Direct inhibition of macropinocytosis causes a morphology change reminiscent of treatment with Berzosertib, including increased vacuolisation and a neurite retraction defect. B) G7

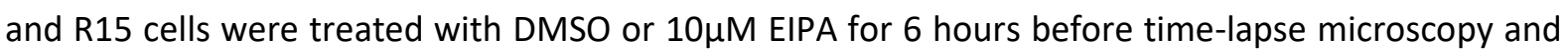
single cell tracking. Data from 3 biological repeats. $* * p>0.01$. 
A
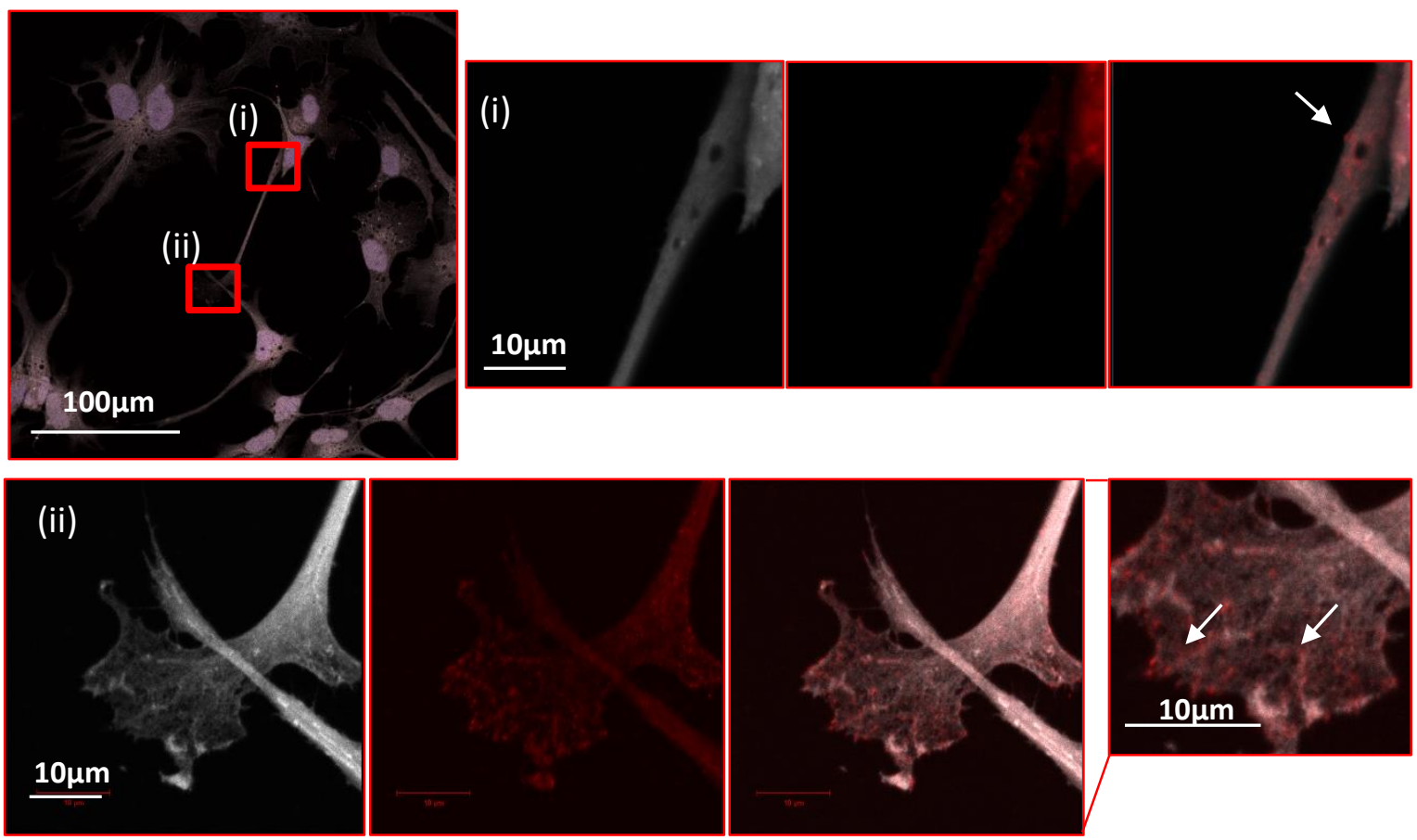

B
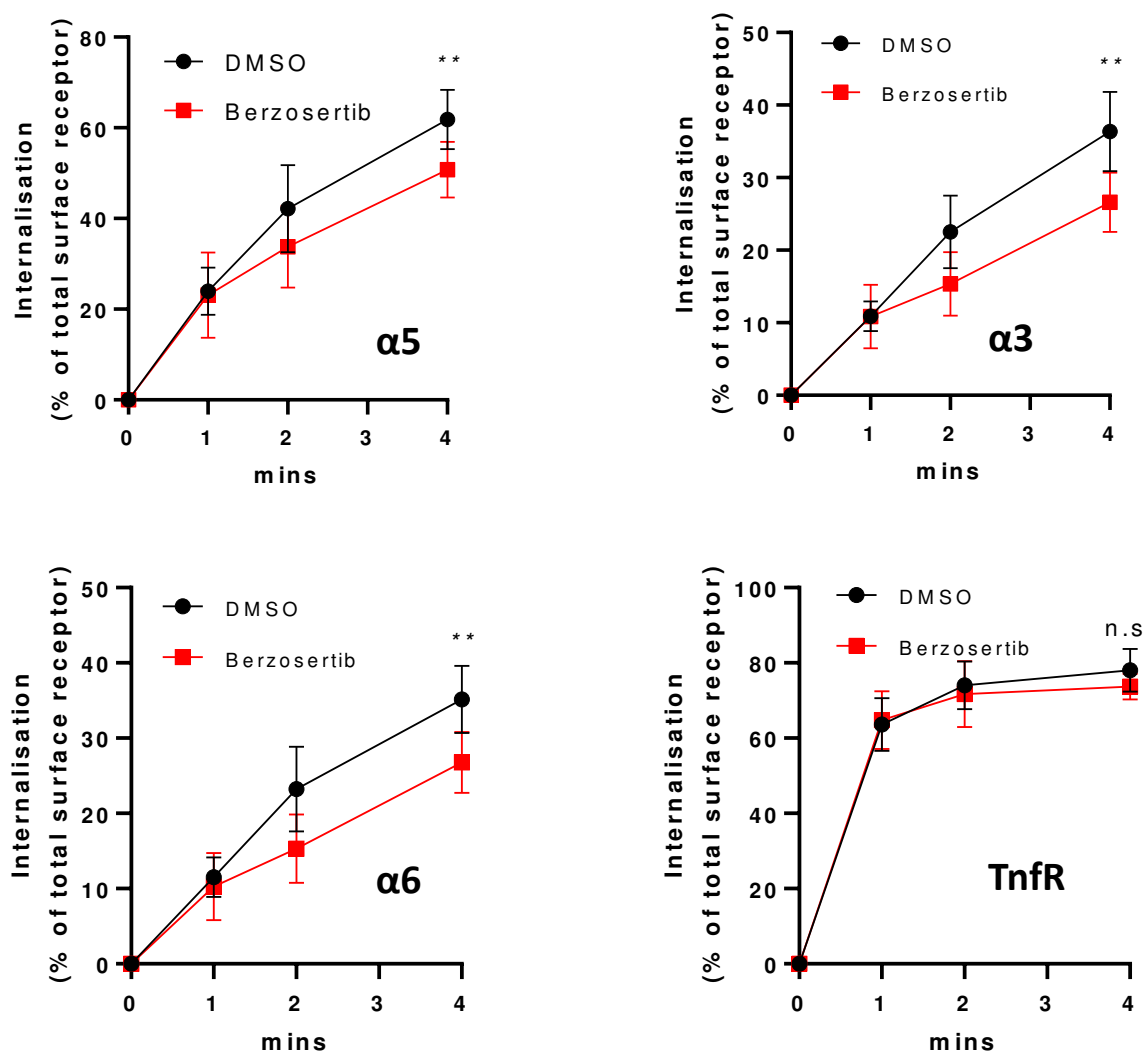
Figure 7: Inhibition of ATR disrupts internalisation of integrins. A) Integrins are found in accumulated on macropinosomal structures after treatment with ATR inhibitor in the cell body (i), and growth conelike structures (ii). E2 cells were treated with DMSO or Berzosertib for 5 hours, followed by fixing and staining for WCS (white), Integrin a6 (red). B) G7 cells were treated with DMSO or Berzosertib before lysis and measurement of internalised pool of integrins. Data from 8 biological repeats over 3 independent experiments. ${ }^{* *} p>0.01$, Mann-whitney 
Birch et al; Fig. 8

A

(i)
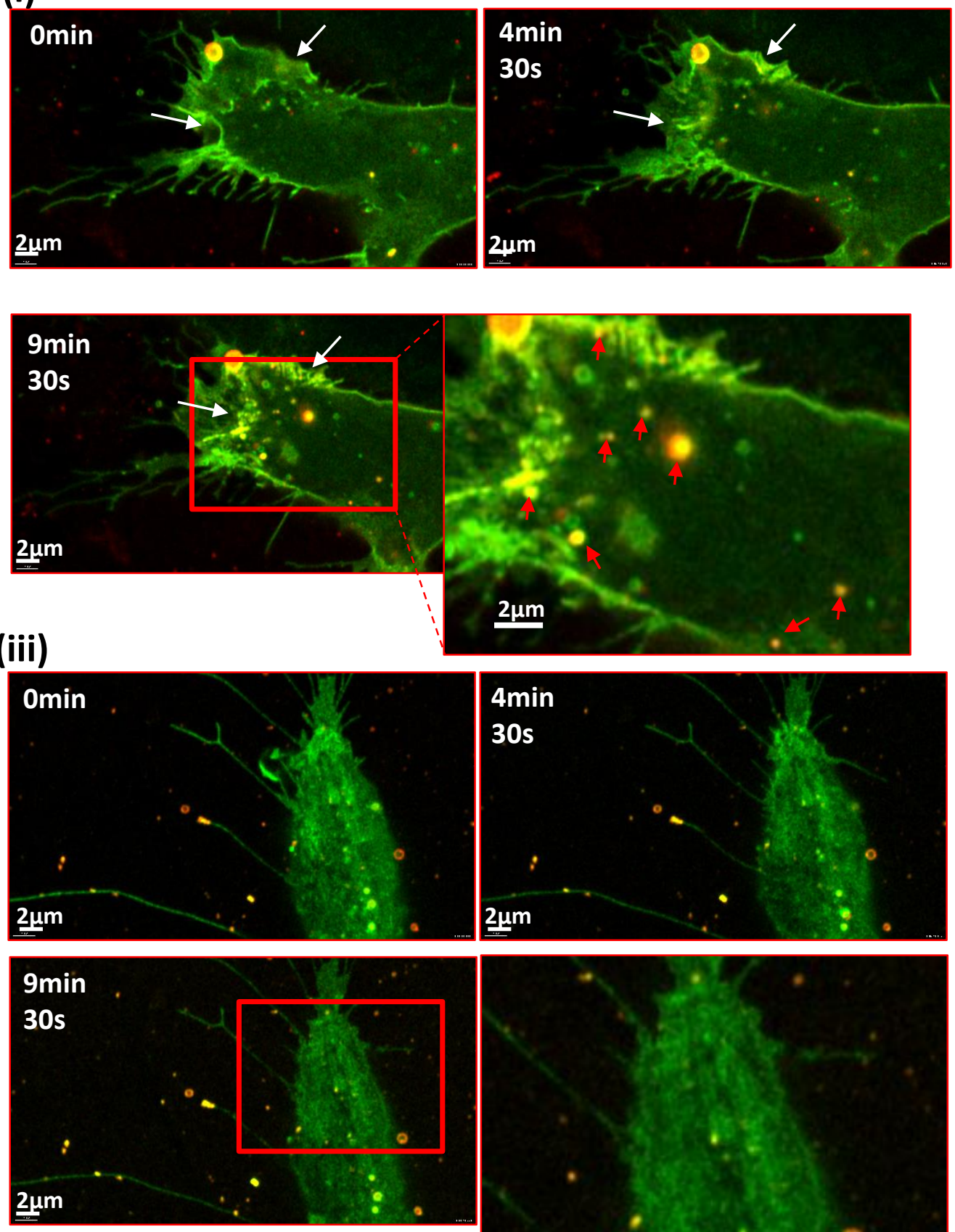
Figure 8: Uptake of integrins via macropinocytosis occurs at growth cones-like structures during neurite retraction (ii) and is inhibited by berzosertib treatment (ii). G7 cells expressing GFP- $\alpha 5$ integrin (green). Cells were incubated with 70 kDaTexas red dextran (red) and DMSO (i) or VE822 (ii) prior to super resolution, time-lapse imaging of growth cones. Yellow $=$ areas of colocalization. White arrows indicate areas of membrane retraction; red arrows indicate dextran positive macropinosomes. 
Birch et al; Fig. S1

A
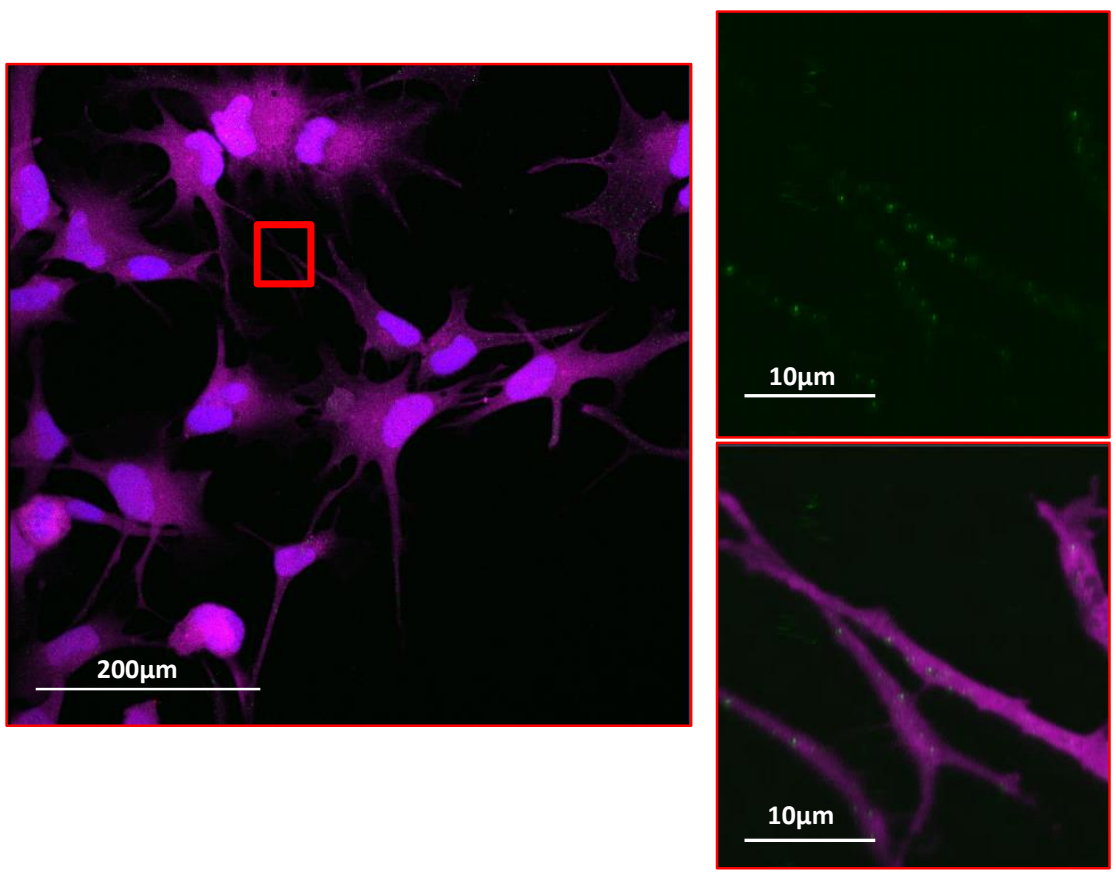

B

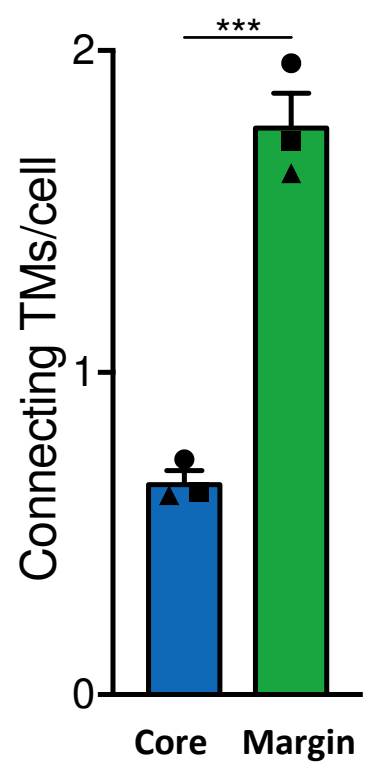

Figure S1 A: E2 cells were fixed and stained for ATR, WCS and DAPI before confocal imaging. B: Number of connecting TM/cell were counted for each cell line. $\mathrm{N}=3,{ }^{* * *} p<0.001$; Students TTest 
Birch et al; Fig. S2

A

E2
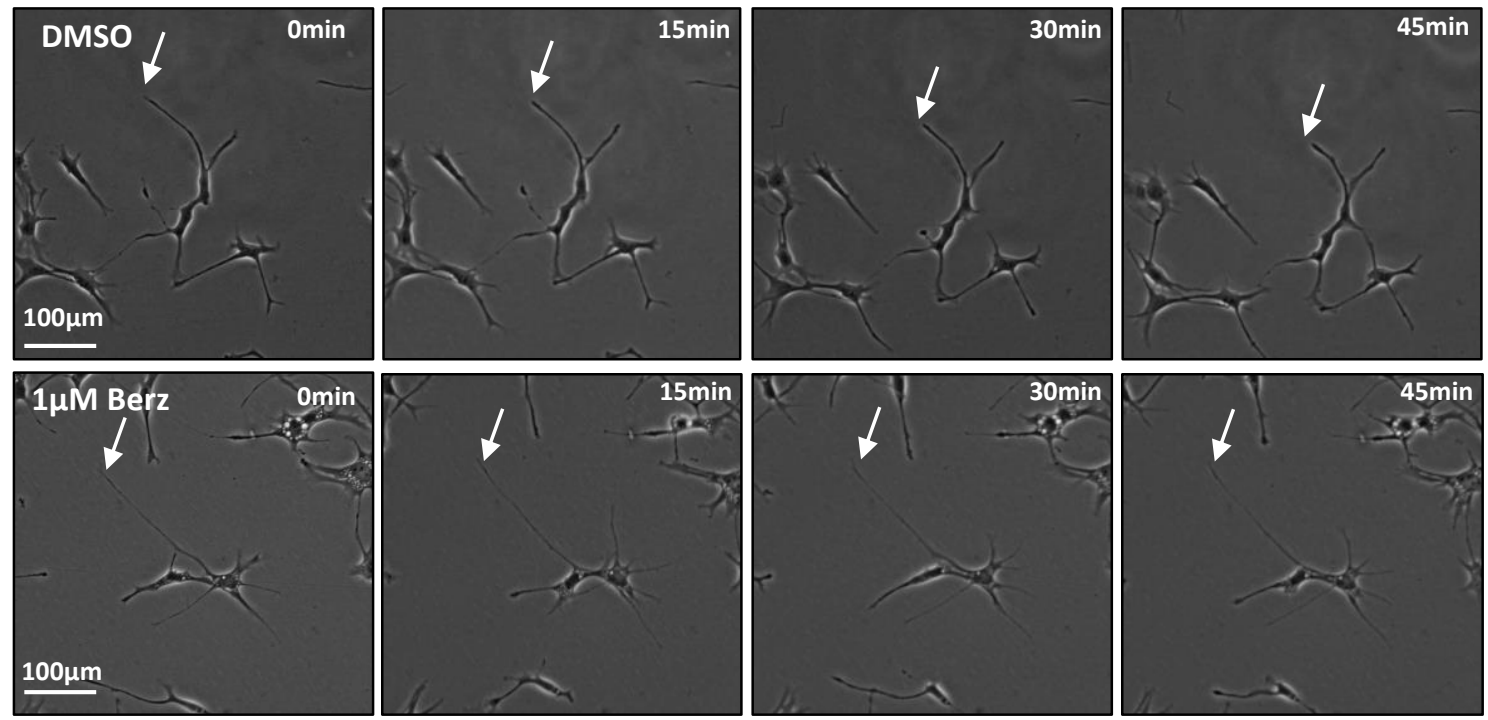

B: Inhibition of ATR induces a neurite retraction defect in E2 cells 
Birch et al; Fig S3

A

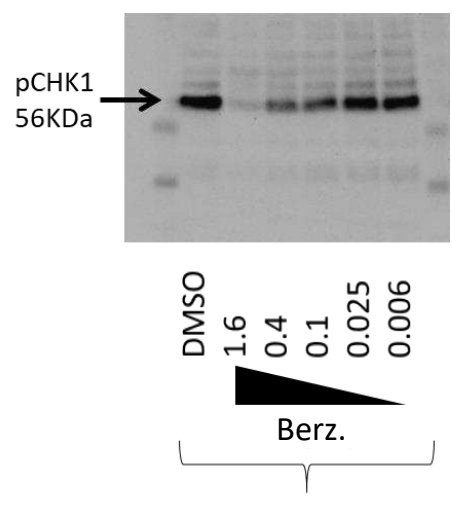

$5 G y$

Figure S2: Western blot for the downstream target of ATR pCHK1 with increasing doses of berzosertib. 
A
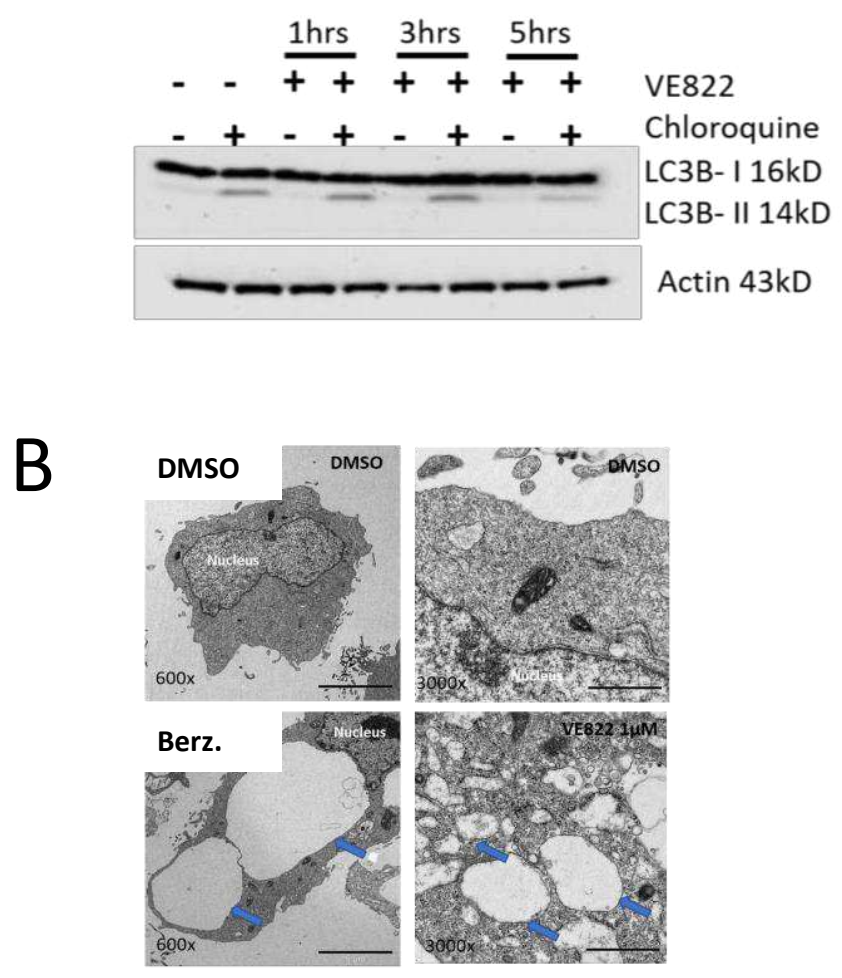

A Western blot analysis for LC3B following treatment with Berzosertib +/- chloroquine and EM imaging demonstrating single membrane encapsulation (B) indicated that the vacuoles are not autophagosomes. 


\section{Supplementary Files}

This is a list of supplementary files associated with this preprint. Click to download.

- SV1.avi

- SV2.avi

- SV3.avi

- NCOMMS2140703reportingsummary.pdf 